Pacific

Journal of

Mathematics

\title{
GENERALIZED EIGENVALUE PROBLEMS OF NONHOMOGENEOUS ELLIPTIC OPERATORS AND THEIR APPLICATION
}

Dumitru Motreanu And Mieko Tanaka

Volume $265 \quad$ No. 1

September 2013 


\title{
GENERALIZED EIGENVALUE PROBLEMS OF NONHOMOGENEOUS ELLIPTIC OPERATORS AND THEIR APPLICATION
}

\author{
Dumitru MOTREANU AND Mieko TANAKA
}

\begin{abstract}
We consider the equation $-\operatorname{div}(a(x,|\nabla u|) \nabla u)=\lambda|u|^{p-2} u$ (whose special case $a(x, t)=t^{p-2}$ is the $p$-Laplace equation) on a bounded domain $\Omega \subset \mathbb{R}^{N}$ with $C^{2}$ boundary, with null boundary condition. We prove that there are $\lambda \in \mathbb{R}$ for which the equation has a nontrivial solution. As an application, by variational methods, we present the existence of a positive solution to $-\operatorname{div}(a(x,|\nabla u|) \nabla u)=f(x, u)$ in $\Omega$, where $f$ is asymptotically $(p-1)$ linear near zero and $\infty$, considering the nonresonant, resonant, and doubly resonant cases. We show that, generally, the spectrum of the operator $-\operatorname{div}(a(x,|\nabla u|) \nabla u)$ on $W_{0}^{1, p}(\Omega)$ is not discrete.
\end{abstract}

\section{Introduction}

Let $1<p<\infty$ and let $\Omega \subset \mathbb{R}^{N}$ be a bounded domain with $C^{2}$ boundary $\partial \Omega$. We are interested in values of $\lambda \in \mathbb{R}$ such that a nontrivial solution exists to the equation

$(\mathrm{EV} ; \lambda)$

$$
\begin{cases}-\operatorname{div} A(x, \nabla u)=\lambda|u|^{p-2} u & \text { in } \Omega, \\ u=0 & \text { on } \partial \Omega\end{cases}
$$

such a $\lambda$ is called an eigenvalue for $A$. Here $A: \bar{\Omega} \times \mathbb{R}^{N} \rightarrow \mathbb{R}^{N}$ is a map that is strictly monotone in the second variable and satisfies the regularity conditions in Assumption A below.

The $p$-Laplace equation is the special case of $(\mathrm{EV} ; \lambda)$ with $A(x, y)=|y|^{p-2} y$, and in this case the eigenvalues for $A$ are the usual eigenvalues of the $p$-Laplacian. However, we do not suppose that $A$ is $(p-1)$-homogeneous in the second variable. Instead, these are the assumptions we make on the map $A$ :

Assumption A. $A(x, y)=a(x,|y|) y$, where $a(x, t)>0$ for all $x \in \bar{\Omega}$ and all $t \in(0,+\infty)$; furthermore:

(i) $A \in C^{0}\left(\bar{\Omega} \times \mathbb{R}^{N}, \mathbb{R}^{N}\right) \cap C^{1}\left(\bar{\Omega} \times\left(\mathbb{R}^{N} \backslash\{0\}\right), \mathbb{R}^{N}\right)$.

MSC2010: 35P30, 35J62, 49R05.

Keywords: quasilinear elliptic equations, nonhomogeneous operators, nonlinear eigenvalue problems, positive solutions, mountain pass theorem. 
(ii) There exists $C_{1}>0$ such that

$$
\left|D_{y} A(x, y)\right| \leq C_{1}|y|^{p-2} \quad \text { for every } x \in \bar{\Omega} \text { and } y \in \mathbb{R}^{N} \backslash\{0\} .
$$

(iii) There exists $C_{0}>0$ such that

$D_{y} A(x, y) \xi \cdot \xi \geq C_{0}|y|^{p-2}|\xi|^{2} \quad$ for every $x \in \bar{\Omega}, y \in \mathbb{R}^{N} \backslash\{0\}$ and $\xi \in \mathbb{R}^{N}$;

(iv) there exists $C_{2}>0$ such that

$$
\left|D_{x} A(x, y)\right| \leq C_{2}\left(1+|y|^{p-1}\right) \quad \text { for every } x \in \bar{\Omega} \text { and } y \in \mathbb{R}^{N} \backslash\{0\} .
$$

(v) There exist $C_{3}>0$ and a positive $t_{0} \leq 1$ such that

$$
\left|D_{x} A(x, y)\right| \leq C_{3}|y|^{p-1}(-\log |y|)
$$

for every $x \in \bar{\Omega}, y \in \mathbb{R}^{N}$ with $0<|y|<t_{0}$.

From now on, we assume that $C_{0} \leq p-1 \leq C_{1}$ which leads to no loss of generality, as can be seen from Assumption A(ii)-(iii).

A similar hypothesis to Assumption A is considered in the study of quasilinear elliptic problems; see [Motreanu and Papageorgiou 2011, Example 2.2; Damascelli 1998; Motreanu et al. 2011; Miyajima et al. 2012; Tanaka 2012a]. We also refer to [García-Huidobro et al. 1995; Kim 2009; Kim and Kim 2010; Fukagai and Narukawa 2007; Prado and Ubilla 1998; Robinson 2004] for generalized $p$-Laplace operators. In particular, when $A(x, y)=|y|^{p-2} y$ - that is, when $\operatorname{div} A(x, \nabla u)$ is the usual $p$-Laplacian $\Delta_{p} u$ - we can take $C_{0}=C_{1}=p-1$ in Assumption A. Conversely, if $C_{0}=C_{1}=p-1$ in Assumption A, the inequalities in Remark 1(ii)-(iii) below show that $a(x, t)=|t|^{p-2}$, whence $A(x, y)=|y|^{p-2} y$. In the $p$-Laplace case, the first eigenvalue $\lambda_{1}$ is obtained by the Rayleigh quotient: $\lambda_{1}=\inf \left\{\int_{\Omega}|\nabla u|^{p} d x /\|u\|_{p}^{p}: u \neq 0\right\}$. But since our operator is nonhomogeneous, $\inf \{\lambda \in \mathbb{R}: \lambda$ is an eigenvalue of $A\}$ is in general not obtained by such a Rayleigh quotient corresponding to $A$. In Section 3 , since the Rayleigh quotient plays an important role, we study its behavior as $\|u\|_{p} \rightarrow 0$ or $\|u\|_{p} \rightarrow \infty$ under an additional condition describing an asymptotic $(p-1)$-homogeneity. For example, we can consider

$$
\operatorname{div} A(x, \nabla u)=\operatorname{div}\left(\left(a_{0}(x)|\nabla u|^{p-2}+a_{\infty}(x)|\nabla u|^{q-2}\right)\left(1+|\nabla u|^{q}\right)^{(p-q) / q} \nabla u\right)
$$

for $1<p \leq q<\infty, a_{0}, a_{\infty} \in C^{1}(\bar{\Omega})$ with $\min _{\bar{\Omega}} a_{0}>0$ and $\min _{\bar{\Omega}} a_{\infty}>0$. This satisfies

$$
\begin{aligned}
A(x, y)-a_{0}(x)|y|^{p-2} y & =o\left(|y|^{p-1}\right) & & \text { as }|y| \rightarrow 0, \\
A(x, y)-a_{\infty}(x)|y|^{p-2} y & =o\left(|y|^{p-1}\right) & & \text { as }|y| \rightarrow \infty .
\end{aligned}
$$

Under these these conditions (see (AH0) and (AH) in Section 3), we shall prove 
that

$$
\min \left\{\int_{\Omega} \int_{0}^{|\nabla u(x)|} \frac{a(x, t) t}{r^{p}} d t d x:\|u\|_{p}=r\right\}
$$

approaches $\lambda_{1}\left(a_{0}\right) / p$ as $r \rightarrow+0$ and $\lambda_{1}\left(a_{\infty}\right) / p$ as $r \rightarrow+\infty$; here

$$
\begin{gathered}
\lambda_{1}\left(a_{0}\right)=\min \left\{\int_{\Omega} a_{0}(x)|\nabla u|^{p} d x:\|u\|_{p}=1\right\}, \\
\lambda_{1}\left(a_{\infty}\right)=\min \left\{\int_{\Omega} a_{\infty}(x)|\nabla u|^{p} d x:\|u\|_{p}=1\right\} .
\end{gathered}
$$

Concerning the eigenvalue problem for a nonhomogeneous operator, we can refer to [Robinson 2004; Tanaka 2012b] under the Neumann boundary condition.

In Section 4, as an application of Section 3, we present the existence of a positive solution for the quasilinear elliptic equation

$$
\begin{cases}-\operatorname{div} A(x, \nabla u)=f(x, u) & \text { in } \Omega, \\ u=0 & \text { on } \partial \Omega,\end{cases}
$$

where $f$ satisfies the following assumption.

Assumption $(\boldsymbol{f}), f$ is a Carathéodory function on $\Omega \times \mathbb{R}$ with $f(x, 0)=0$ for a.e. $x \in \Omega, f$ is bounded on bounded sets and $f$ is asymptotically ( $p-1)$-linear near +0 and $+\infty$ in the following sense:

$$
\begin{array}{ll}
\lim _{u \rightarrow+0} \frac{f(x, u)}{u^{p-1}}=\alpha_{0} & \text { uniformly in a.e. } x \in \Omega, \\
\lim _{u \rightarrow+\infty} \frac{f(x, u)}{u^{p-1}}=\alpha & \text { uniformly in a.e. } x \in \Omega,
\end{array}
$$

for some constants $\alpha_{0}$ and $\alpha$.

Regarding the existence of a positive solution under the Dirichlet boundary condition, we can refer to [Fukagai and Narukawa 2007; Prado and Ubilla 1998] for nonhomogeneous operators. However, we can not apply these results to our nonlinear term which is only asymptotically $(p-1)$-linear near +0 and $+\infty$, and furthermore with possibly different weights. In [García-Huidobro et al. 1995], it is proved the existence of a positive radial solution for nonhomogeneous operators.

For the $p$-Laplace equation, it is well known that if $\left(\alpha-\lambda_{1}\right)\left(\alpha_{0}-\lambda_{1}\right)<0$ (where $\lambda_{1}$ denotes the first eigenvalue of $-\Delta_{p}$ under a Dirichlet boundary condition),

$$
-\Delta_{p} u=f(x, u) \quad \text { in } \Omega, \quad u=0 \quad \text { on } \partial \Omega,
$$

has a positive solution (see [Dancer and Perera 2001]). One of our main purposes is to extend this existence result from the $p$-Laplace equation to the corresponding problem involving our nonhomogeneous operator $A$. This is done in Theorem 25 . We mention that in the special case of $A(x, y)=A(y)$, the result in [Kyritsi 
et al. 2010] provides the existence of a positive solution if $\alpha<\lambda_{1} C_{0} /(p-1)$ and $\lambda_{1} C_{1} /(p-1)<\alpha_{0}$ hold (note that we can apply this result only to the case where $\left.\alpha<\alpha_{0}\right)$. We emphasize that, for our general operator, the case $\lambda_{1}\left(a_{0}\right) \neq \lambda_{1}\left(a_{1}\right)$ can occur. Note that in such a situation, contrary to the $p$-Laplacian case, we can still apply our theorem when $\alpha_{0}=\alpha$ provided this number is between $\lambda_{1}\left(a_{0}\right)$ and $\lambda_{1}\left(a_{1}\right)$. The known result for the $p$-Laplacian case is obtained from our theorem simply by setting $a_{0} \equiv 1$ and $a_{\infty} \equiv 1$.

In particular, our theorem implies that if $\lambda_{1}\left(a_{0}\right) \neq \lambda_{1}\left(a_{\infty}\right)$, then every $\lambda$ between $\lambda_{1}\left(a_{0}\right)$ and $\lambda_{1}\left(a_{\infty}\right)$ is an eigenvalue of $A$ (see Corollary 26) and has a positive eigenfunction. This shows that, generally, the spectrum of the operator $-\operatorname{div} A(x, \nabla \cdot)$ on $W_{0}^{1, p}(\Omega)$ is not discrete.

In the final part of the paper, we treat the one side resonant and doubly resonant cases under additional conditions on $f$. For the $p$-Laplace equation, we refer to [Tanaka 2009] for the resonant and doubly resonant cases. Our Theorem 31 provides the existence of a positive solution in all cases of resonance for problem $(\mathrm{P})$ with a nonhomogeneous operator in the principal part.

\section{The properties of the map $A$}

In what follows, the norm on $W_{0}^{1, p}(\Omega)$ is given by

$$
\|u\|^{p}:=\|\nabla u\|_{p}^{p},
$$

where $\|u\|_{q}$ denotes the usual norm of $L^{q}(\Omega)$ for $u \in L^{q}(\Omega)(1 \leq q \leq \infty)$. Setting

$$
G(x, y):=\int_{0}^{|y|} a(x, t) t d t
$$

we can easily see that

$$
\nabla_{y} G(x, y)=A(x, y) \text { and } G(x, 0)=0
$$

for every $x \in \bar{\Omega}$; see [Motreanu et al. 2011] for details.

Remark 1. The following assertions hold under Assumption A:

(i) For all $x \in \bar{\Omega}, A(x, y)$ is maximal monotone and strictly monotone in $y$.

(ii) $|A(x, y)| \leq \frac{C_{1}}{p-1}|y|^{p-1}$ for every $(x, y) \in \bar{\Omega} \times \mathbb{R}^{N}$.

(iii) $A(x, y) y \geq \frac{C_{0}}{p-1}|y|^{p}$ for every $(x, y) \in \bar{\Omega} \times \mathbb{R}^{N}$.

(iv) $G(x, y)$ is strictly convex in $y$ for all $x$ and satisfies the inequalities

(2) $\quad A(x, y) y \geq G(x, y) \geq \frac{C_{0}}{p(p-1)}|y|^{p} \quad$ and $\quad G(x, y) \leq \frac{C_{1}}{p(p-1)}|y|^{p}$ for every $(x, y) \in \bar{\Omega} \times \mathbb{R}^{N}$. 
The following result is important for the proof of the Palais-Smale condition for the functionals related to our problem.

Proposition 2 [Motreanu et al. 2011, Proposition 1]. Let $V: W_{0}^{1, p}(\Omega) \rightarrow W_{0}^{1, p}(\Omega)^{*}$ be the map defined by

$$
\langle V(u), v\rangle=\int_{\Omega} A(x, \nabla u) \nabla v d x
$$

for $u, v \in W_{0}^{1, p}(\Omega)$. Then any sequence $\left\{u_{m}\right\}$ that converges weakly to $u$ and satisfies $\lim _{\sup _{m \rightarrow \infty}}\left\langle V\left(u_{m}\right), u_{m}-u\right\rangle \leq 0$ also converges strongly to $u$.

Remark 3. (i) If $u \in W_{0}^{1, p}(\Omega)$ is a solution of $(\mathrm{P})$, then $u \in C^{1, \alpha}(\bar{\Omega})$ for some $0<\alpha<1$.

(ii) If $u \in W_{0}^{1, p}(\Omega)$ is a nontrivial solution of $(\mathrm{P})$ such that $u \geq 0$, then $u>0$ in $\Omega$ and $\partial u / \partial v<0$ on $\partial \Omega$, where $v$ denotes the outward unit normal vector on $\partial \Omega$.

Sketch of proof. (i) Let $u \in W_{0}^{1, p}(\Omega)$ be a solution of (P). Then, because $u \in L^{\infty}(\Omega)$ as shown by using the Moser iteration process (cf. [Miyajima et al. 2012, Appendix]), we see that $u \in C^{1, \alpha}(\bar{\Omega})(0<\alpha<1)$ by the regularity result in [Lieberman 1988].

(ii) Let $u \in W_{0}^{1, p}(\Omega)$ be a solution of (P) satisfying $u \geq 0$ and $u \neq \equiv$. Then, by Assumption $(f)$, we obtain a constant $\lambda>0$ satisfying

$$
-\operatorname{div} A(x, \nabla u)+\lambda u^{p-1} \geq 0 \quad \text { in } \Omega .
$$

Noting that $u \in C^{1, \alpha}(\bar{\Omega})(0<\alpha<1)$ by (i), we have $u(x)>0$ for every $x \in \Omega$ by [Miyajima et al. 2012, Appendix, Theorem B]. In addition, using the strong maximum principle [ibid., Appendix, Theorem A], we easily see that $\partial u(x) / \partial v<0$ for every $x \in \partial \Omega$.

Proposition 4. Let $f_{n}: \Omega \times \mathbb{R} \rightarrow \mathbb{R}$ be a Carathéodory function satisfying

$$
\left|f_{n}(x, t)\right| \leq D\left(1+|t|^{r-1}\right) \quad \text { for every } x \in \Omega, t \in \mathbb{R}
$$

with some positive constant $D$ independent of $n$ and $r \in\left[p, p^{*}\right)$, where $p^{*}=\infty$ if $N \leq p$ and $p^{*}=p N /(N-p)$ if $N>p$. Assume that $A_{n}: \Omega \times \mathbb{R}^{N} \rightarrow \mathbb{R}^{N}$ is a map satisfying parts (i)-(iv) of Assumption A with positive constants $C_{1}^{\prime}, C_{0}^{\prime}$, and $C_{2}^{\prime}$ independent of $n$. If $u_{n}$ is a solution for

$$
-\operatorname{div} A_{n}(x, \nabla u)=f_{n}(x, u) \quad \text { in } \Omega, \quad u=0 \quad \text { on } \partial \Omega
$$

and $\left\{u_{n}\right\}$ is bounded in $W_{0}^{1, p}(\Omega)$, then there exist a subsequence $\left\{u_{n_{l}}\right\}$ of $\left\{u_{n}\right\}$ and $u_{0} \in C_{0}^{1}(\bar{\Omega})$ such that $u_{n_{l}} \rightarrow u_{0}$ in $C_{0}^{1}(\bar{\Omega})$ as $l \rightarrow \infty$.

Proof. Since $\left\{u_{n}\right\}$ is bounded in $W_{0}^{1, p}(\Omega)$, we may assume that $u_{n}$ converges weakly to some $u_{0}$ in $W_{0}^{1, p}(\Omega)$ by choosing a subsequence. We can show that there exists a $C>0$ depending only on $|\Omega|, p, N, D, C_{0}^{\prime}, C_{1}^{\prime}$, and the embedding constant of 
$W_{0}^{1, p}(\Omega)$ into $L^{\bar{p}^{*}}(\Omega)$ such that $\left\|u_{n}\right\|_{\infty} \leq C \max \left\{1,\left\|u_{n}\right\|^{\left(\bar{p}^{*}-p\right) /\left(\bar{p}^{*}-r\right)}\right\}$ by the Moser iteration process to [Miyajima et al. 2012, Theorem C], where $\bar{p}^{*}=p^{*}$ if $N>p$ and $\bar{p}^{*}>r$ is any constant if $N \leq p$. Since $D, C_{1}^{\prime}$, and $C_{0}^{\prime}$ are independent of $n$, $\left\|u_{n}\right\|_{\infty}$ is bounded. Therefore, the regularity result in [Lieberman 1988] guarantees that there exist $\gamma \in(0,1)$ and $M>0$ independent of $n$ such that $u_{n} \in C_{0}^{1, \gamma}(\bar{\Omega})$ and $\left\|u_{n}\right\|_{C_{0}^{1, \gamma}}(\bar{\Omega}) \leq M$ (where we use the fact that $C_{2}^{\prime}$ is independent of $n$ ). Since the inclusion of $C_{0}^{1, \gamma}(\bar{\Omega})$ to $C_{0}^{1}(\bar{\Omega})$ is compact, $u_{n}$ converges to $u_{0}$ in $C_{0}^{1}(\bar{\Omega})$ (note that $u_{n} \rightarrow u_{0}$ in $\left.W_{0}^{1, p}(\Omega)\right)$.

\section{Eigenvalue problems}

We introduce a function $J: W_{0}^{1, p}(\Omega) \rightarrow \mathbb{R}$ by

$$
J(u)=\int_{\Omega} G(x, \nabla u) d x \quad \text { for all } u \in W_{0}^{1, p}(\Omega) .
$$

It is clear that $J$ is of class $C^{1}$. We also note that

$$
r S:=\left\{u \in W_{0}^{1, p}(\Omega):\|u\|_{p}=r\right\} \text { for } r>0
$$

is a $C^{1}$ Finsler manifold (cf. [Deimling 1985, Sections 27.4 and 27.5]) because $r$ is a regular value of the function $u \mapsto\|u\|_{p}$ on $W_{0}^{1, p}(\Omega)$. Hence the norm of the derivative at $u \in(r S)$ of the restriction $\tilde{J}$ of $J$ to $r S$ is defined by

$$
\begin{aligned}
\left\|\tilde{J}^{\prime}(u)\right\|_{*} & :=\min \left\{\left\|J^{\prime}(u)-t \Phi^{\prime}(u)\right\|_{W_{0}^{1, p}(\Omega)^{*}}: t \in \mathbb{R}\right\} \\
& =\sup \left\{\left\langle J^{\prime}(u), v\right\rangle: v \in T_{u}(r S),\|v\|=1\right\},
\end{aligned}
$$

where $\Phi(u):=(1 / p)\|u\|_{p}^{p}$ and $T_{u}(r S)$ denotes the tangent space of $r S$ at $u$, that is, $T_{u}(r S)=\left\{v \in W_{0}^{1, p}(\Omega): \int_{\Omega}|u|^{p-2} u v d x=0\right\}$. It follows that the restriction $\tilde{J}=\left.J\right|_{(r S)}$ is a $C^{1}$-function on $r S$ in the sense of manifolds.

Proposition 5. For $r>0$, the infimum

$$
\mu_{1}(A, r)=\inf _{u \in(r S)} \int_{\Omega} G(x, \nabla u) d x
$$

is attained at points $\pm \hat{u}_{r} \in(r S)$ with $\hat{u}_{r} \in C^{1, \alpha}(\bar{\Omega})$ and $\hat{u}_{r}>0$ in $\Omega$. Moreover, $\pm \hat{u}_{r}$ are solutions of $(\mathrm{EV} ; \lambda)$ with $\lambda=\lambda_{1}\left(A, \hat{u}_{r}\right) / r^{p}$, where

$$
\lambda_{1}\left(A, \hat{u}_{r}\right)=\int_{\Omega} A\left(x, \nabla \hat{u}_{r}\right) \nabla \hat{u}_{r} d x \geq \frac{C_{0}}{p-1} \lambda_{1} r^{p} .
$$

Proof. Let $\left\{u_{n}\right\} \subset(r S)$ be a minimizing sequence for (5). Using (2), it follows that $\left\{u_{n}\right\}$ is bounded in $W_{0}^{1, p}(\Omega)$, so along a relabeled subsequence we have $u_{n} \rightarrow u$ in $W_{0}^{1, p}(\Omega)$ and $u_{n} \rightarrow u$ in $L^{p}(\Omega)$ for some $u \in W_{0}^{1, p}(\Omega)$, thus $u \in(r S)$. Since 
$G(x, \cdot)$ is convex and continuous for all $x \in \Omega, J$ is weakly lower semicontinuous on $W_{0}^{1, p}(\Omega)$ [Mawhin and Willem 1989, Theorem 1.2]. Therefore, we derive that

$$
\mu_{1}(A, r) \leq \int_{\Omega} G(x, \nabla u) d x \leq \liminf _{n \rightarrow \infty} \int_{\Omega} G\left(x, \nabla u_{n}\right) d x,
$$

which yields

$$
\mu_{1}(A, r)=\int_{\Omega} G(x, \nabla u) d x .
$$

The fact that the functional $J$ is even implies that $|u|$ is also a global minimizer of $\tilde{J}_{r}$. Consequently, we may assume that $u \geq 0$. On the other hand, the Lagrange multiplier rule leads to the existence of $t \in \mathbb{R}$ such that

$$
\int_{\Omega} A(x, \nabla u) \nabla v d x=t \int_{\Omega} u^{p-1} v d x \quad \text { for all } v \in W_{0}^{1, p}(\Omega) .
$$

Inserting $v=u$ in (7) entails

$$
\operatorname{tr}^{p}=\int_{\Omega} A(x, \nabla u) \nabla u d x \geq \frac{C_{0}}{p-1}\|\nabla u\|_{p}^{p} \geq \frac{C_{0} \lambda_{1}}{p-1}\|u\|_{p}^{p}=\frac{C_{0} \lambda_{1}}{p-1} r^{p} .
$$

Therefore, we have

$$
t=\frac{\lambda_{1}(A, u)}{r^{p}} \geq \frac{C_{0} \lambda_{1}}{p-1} .
$$

From (7), it follows that $u$ is a solution of $(\mathrm{EV} ; \lambda)$ with $\lambda=t=\lambda_{1}(A, u) / r^{p}$. According to Remark 3 with $f(x, u)=t|u|^{p-2} u$, it follows that $u \in C^{1, \alpha}(\bar{\Omega})$ $(0<\alpha<1)$ and $u>0$ in $\Omega$. Since $J$ is even and $\lambda_{1}(A, u)=\lambda_{1}(A,-u)$, we have that $J(-u)=J(u)=\mu_{1}(A, r)$ and $-u$ is a negative solution of $(\mathrm{EV} ; \lambda)$ with $\lambda=t=\lambda_{1}(A, u) / r^{p}$. The result is thus established with $\hat{u}_{r}=u$.

We define

$$
K_{1}(A, r):=\left\{u \in(r S): J(u)=\mu_{1}(A, r)\right\} .
$$

Then it follows from Proposition 5 that $K_{1}(A, r)$ is not empty for each $r>0$.

Because we do not know whether the minimizers of $\tilde{J}_{r}$ are only $\pm \hat{u}_{r}$, we introduce the following:

$$
\begin{aligned}
& \underline{\lambda}_{1}(A, r):=\inf \left\{\int_{\Omega} A(x, \nabla u) \nabla u d x: u \in K_{1}(A, r)\right\}, \\
& \bar{\lambda}_{1}(A, r):=\sup \left\{\int_{\Omega} A(x, \nabla u) \nabla u d x: u \in K_{1}(A, r)\right\} .
\end{aligned}
$$

Lemma 6. For every $r>0, \underline{\lambda}_{1}(A, r)$ and $\bar{\lambda}_{1}(A, r)$ are attained.

Proof. We only deal with $\underline{\lambda}_{1}(A, r)$ because $\bar{\lambda}_{1}(A, r)$ can be treated similarly. Fix any $r>0$. Let $u_{n} \in K_{1}(A, r)$ satisfy $\lambda_{1}\left(A, u_{n}\right) \rightarrow \underline{\lambda}_{1}(A, r)$ as $n \rightarrow \infty$. Then we 
see that $\left\|\nabla u_{n}\right\|_{p}$ is bounded from the inequality

$$
\frac{C_{0}}{p(p-1)}\left\|\nabla u_{n}\right\|_{p}^{p} \leq \int_{\Omega} G\left(x, \nabla u_{n}\right) d x=\mu_{1}(A, r) \leq \int_{\Omega} G(x, \nabla w) d x
$$

for $w \in r S$, where we use the definition of $\mu_{1}(A, r)$ and (2). Recall that each $u_{n}$ is a solution of $(\mathrm{EV} ; \lambda)$ with $\lambda=\lambda_{1}\left(A, u_{n}\right) / r^{p}$. Moreover, we have

$$
\frac{C_{0}}{p-1} \lambda_{1} r^{p} \leq \lambda_{1}\left(A, u_{n}\right) \leq \frac{C_{1}}{p-1}\left\|\nabla u_{n}\right\|_{p}^{p}
$$

by Remark 1(ii) (see (6) for the first inequality), whence $\lambda_{1}\left(A, u_{n}\right) / r^{p}$ is bounded. As a result, due to Proposition 4, we may assume that there exists $u_{0} \in W_{0}^{1, p}(\Omega)$ such that $u_{n} \rightarrow u_{0}$ in $C_{0}^{1}(\bar{\Omega})$ by choosing a subsequence if necessary. Since $J$ and $\lambda_{1}(A, \cdot)$ are continuous in $W_{0}^{1, p}(\Omega)$, we see that $J\left(u_{0}\right)=\lim _{n \rightarrow \infty} J\left(u_{n}\right)=$ $\mu_{1}(A, r), u_{0} \in K_{1}(A, r)$, and $\lambda_{1}\left(A, u_{0}\right)=\lim _{n \rightarrow \infty} \lambda_{1}\left(A, u_{n}\right)=\underline{\lambda}_{1}(A, r)$. Thus, our conclusion holds.

Define

$$
\lambda_{1}(A):=\inf _{u \neq 0} \int_{\Omega} \frac{A(x, \nabla u) \nabla u}{\|u\|_{p}^{p}} d x \quad \text { and } \quad \mu_{1}(A):=\inf _{u \neq 0} \int_{\Omega} \frac{G(x, \nabla u)}{\|u\|_{p}^{p}} d x .
$$

\section{Lemma 7.}

$\frac{C_{0}}{p-1} \lambda_{1} \leq \lambda_{1}(A) \leq \min \left\{\inf _{r>0} \frac{\lambda_{1}(A, r)}{r^{p}}, \frac{C_{1}}{p-1} \lambda_{1}\right\} \quad$ and $\quad \mu_{1}(A)=\inf _{r>0} \frac{\mu_{1}(A, r)}{r^{p}}$.

Proof. First, we consider $\lambda_{1}(A)$. For every $0 \neq u \in W_{0}^{1, p}(\Omega)$, we have

$$
\frac{C_{0}}{p-1} \frac{\|\nabla u\|_{p}^{p}}{\|u\|_{p}^{p}} \leq \int_{\Omega} \frac{A(x, \nabla u) \nabla u}{\|u\|_{p}^{p}} d x \leq \frac{C_{1}}{p-1} \frac{\|\nabla u\|_{p}^{p}}{\|u\|_{p}^{p}}
$$

by Remark 1(ii)-(iii). Thus $\left(C_{0} /(p-1)\right) \lambda_{1} \leq \lambda_{1}(A) \leq\left(C_{1} /(p-1)\right) \lambda_{1}$ by taking the infimum with respect to $u$.

Here we fix any $\varepsilon>0$. Then there exists an $r_{\varepsilon}>0$ such that $\underline{\lambda}_{1}\left(A, r_{\varepsilon}\right) / r_{\varepsilon}^{p} \leq$ $\inf _{r>0}\left(\underline{\lambda}_{1}(A, r) / r^{p}\right)+\varepsilon$. By Lemma 6, we can choose $u_{\varepsilon} \in\left(r_{\varepsilon} S\right)$ such that $\lambda_{1}\left(A, u_{\varepsilon}\right)=\underline{\lambda}_{1}\left(A, r_{\varepsilon}\right)$, that is, $\int_{\Omega} A\left(x, \nabla u_{\varepsilon}\right) \nabla u_{\varepsilon} d x=\underline{\lambda}_{1}\left(A, r_{\varepsilon}\right)$. By the definition of $\lambda_{1}(A)$, we obtain

$$
\lambda_{1}(A) \leq \int_{\Omega} \frac{A\left(x, \nabla u_{\varepsilon}\right) \nabla u_{\varepsilon}}{\left\|u_{\varepsilon}\right\|_{p}^{p}} d x=\frac{\underline{\lambda}_{1}\left(A, r_{\varepsilon}\right)}{r_{\varepsilon}^{p}} \leq \inf _{r>0} \frac{\underline{\lambda}_{1}(A, r)}{r^{p}}+\varepsilon .
$$

Because $\varepsilon>0$ is arbitrary, we have $\lambda_{1}(A) \leq \inf _{r>0}\left(\underline{\lambda}_{1}(A, r) / r^{p}\right)$.

Next we treat $\mu_{1}(A)$. Fix any $\varepsilon>0$. Then there exists an $r_{\varepsilon}>0$ such that $\mu_{1}\left(A, r_{\varepsilon}\right) / r_{\varepsilon}^{p} \leq \inf _{r>0}\left(\mu_{1}(A, r) / r^{p}\right)+\varepsilon$. On the other hand, because $\mu_{1}\left(A, r_{\varepsilon}\right)$ is 
attained at some $u_{\varepsilon} \in\left(r_{\varepsilon} S\right)$, we have

$$
\inf _{u \neq 0} \int_{\Omega} \frac{G(x, \nabla u)}{\|u\|_{p}^{p}} d x \leq \int_{\Omega} \frac{G\left(x, \nabla u_{\varepsilon}\right)}{\left\|u_{\varepsilon}\right\|_{p}^{p}} d x=\frac{\mu_{1}\left(A, r_{\varepsilon}\right)}{r_{\varepsilon}^{p}} \leq \inf _{r>0} \frac{\mu_{1}(A, r)}{r^{p}}+\varepsilon .
$$

Because $\varepsilon>0$ is arbitrary, this yields that $\mu_{1}(A) \leq \inf _{r>0}\left(\mu_{1}(A, r) / r^{p}\right)$.

For any $\varepsilon>0$, we take $v_{\varepsilon} \neq 0$ such that $\int_{\Omega}\left(G\left(x, \nabla v_{\varepsilon}\right) /\left\|v_{\varepsilon}\right\|_{p}^{p}\right) d x \leq \mu_{1}(A)+\varepsilon$. Then $r_{\varepsilon}:=\left\|v_{\varepsilon}\right\|_{p}>0$ and so

$$
\frac{\mu_{1}\left(A, r_{\varepsilon}\right)}{r_{\varepsilon}^{p}} \leq \int_{\Omega} \frac{G\left(x, \nabla v_{\varepsilon}\right)}{\left\|v_{\varepsilon}\right\|_{p}^{p}} d x \leq \mu_{1}(A)+\varepsilon .
$$

This leads to $\mu_{1}(A) \geq \inf _{r>0}\left(\mu_{1}(A, r) / r^{p}\right)$.

Proposition 8. If $\lambda<\lambda_{1}(A)$, (EV; $\left.\lambda\right)$ has no nontrivial solutions.

Proof. Let $u$ be a nontrivial solution of $(\mathrm{EV} ; \lambda)$ with $\lambda<\lambda_{1}(A)$. Then we have

$$
\lambda_{1}(A) \leq \int_{\Omega} \frac{A(x, \nabla u) \nabla u}{\|u\|_{p}^{p}} d x=\lambda
$$

by the definition of $\lambda_{1}(A)$. This is a contradiction.

Set

$$
A_{p}:=\frac{C_{1}}{p-1}\left(\frac{C_{1}}{C_{0}}\right)^{p-1} \geq 1,
$$

which is equal to 1 exactly in the case of $A(x, y)=|y|^{p-2} y$ (that is, the special case of the $p$-Laplacian ) because we can choose $C_{0}=C_{1}=p-1$.

Lemma 9 [Tanaka 2012a, Lemma 16]. Let $\varepsilon>0$. For every

$$
u, \varphi \in W^{1, p}(\Omega) \cap C^{1}(\Omega) \cap L^{\infty}(\Omega)
$$

with $u \geq 0$ and $\varphi \geq 0$ in $\Omega$, we have

$$
\int_{\Omega} A(x, \nabla u) \nabla\left(\frac{\varphi^{p}}{(u+\varepsilon)^{p-1}}\right) d x \leq A_{p}\|\nabla \varphi\|_{p}^{p} .
$$

Proposition 10. Any nontrivial solution of $(\mathrm{EV} ; \lambda)$ with $\lambda>A_{p} \lambda_{1}$ changes sign.

Proof. By way of contradiction, assume there is a solution $u$ that does not change sign. Then we may suppose that $u \geq 0$ because $A$ is odd. Due to the strong maximum principle and the regularity theorem (see Remark 3), it follows that $u \in C_{0}^{1}(\bar{\Omega})$ and $u>0$ in $\Omega$. Let $\varphi_{1}$ be the positive eigenfunction of $-\Delta_{p}$ corresponding to $\lambda_{1}$ such that $\left\|\varphi_{1}\right\|_{p}=1$. According to Lemma 9, we obtain

$A_{p} \lambda_{1}=A_{p}\left\|\nabla \varphi_{1}\right\|_{p}^{p} \geq \int_{\Omega} A(x, \nabla u) \nabla\left(\frac{\varphi_{1}^{p}}{(u+\varepsilon)^{p-1}}\right) d x=\lambda \int_{\Omega}\left(\frac{u}{u+\varepsilon}\right)^{p-1} \varphi_{1}^{p} d x$

for every $\varepsilon>0$. By taking $\varepsilon \downarrow 0$, we have $\lambda \leq A_{p} \lambda_{1}$. This is a contradiction. 
Proposition 11. Assume $A_{p} \lambda_{1}<C_{0} \lambda_{2} /(p-1)$, where $\lambda_{2}>\lambda_{1}$ is the second eigenvalue of $-\Delta_{p}$. If $A_{p} \lambda_{1}<\lambda<C_{0} \lambda_{2} /(p-1)$, (EV; $\left.\lambda\right)$ has no nontrivial solutions.

Proof. By way of contradiction, we assume that $(\mathrm{EV} ; \lambda)$ has a nontrivial solution $u$. Then it follows from Proposition 10 that $u$ changes sign. Moreover, by taking $u_{ \pm}$ as a test function in $(E V ; \lambda)$, we have

$$
\frac{C_{0}}{p-1}\left\|\nabla u_{ \pm}\right\|_{p}^{p} \leq \int_{\Omega} A(x, \nabla u)\left( \pm \nabla u_{ \pm}\right) d x=\lambda\left\|u_{ \pm}\right\|_{p}^{p},
$$

whence

$$
\left\|\nabla u_{ \pm}\right\|_{p}^{p}<\lambda_{2}\left\|u_{ \pm}\right\|_{p}^{p} .
$$

This inequality guarantees the existence of a continuous path $\gamma_{0}$ on $S$ such that $\gamma_{0}(0)=\varphi_{1}, \gamma_{0}(1)=-\varphi_{1}$ and $\max _{t \in[0,1]}\left\|\nabla \gamma_{0}(t)\right\|_{p}^{p}<\lambda_{2}$; refer to [Cuesta et al. 1999, Lemma 5.3]. This contradicts the equality

$$
\lambda_{2}=\inf _{\gamma \in \Sigma} \max _{t \in[0,1]} \Phi(\gamma(t)),
$$

where $\Phi(u):=\|\nabla u\|_{p}^{p}$ and $\Sigma:=\left\{\gamma \in C([0,1], S): \gamma(0)=\varphi_{1}, \gamma(1)=-\varphi_{1}\right\}$; see [Anane 1987; Cuesta et al. 1999]. This contradiction proves our result.

For the reader's convenience, we give the sketch of the construction of a path $\gamma_{0}$ as required above. Define paths as follows:

$$
\begin{aligned}
& \gamma_{1}(t):=\frac{t u+(1-t) u_{+}}{\left\|t u+(1-t) u_{+}\right\|_{p}}=\frac{u_{+}-t u_{-}}{\left\|u_{+}-t u_{-}\right\|_{p}}, \quad \gamma_{2}(t):=\frac{t u_{+}+(1-t) u_{-}}{\left\|t u_{+}+(1-t) u_{-}\right\|_{p}}, \\
& \gamma_{3}(t):=\frac{(1-t) u-t u_{-}}{\left\|(1-t) u-t u_{-}\right\|_{p}}=\frac{(1-t) u_{+}-u_{-}}{\left\|(1-t) u_{+}-u_{-}\right\|_{p}}
\end{aligned}
$$

for $t \in[0,1]$. Then, setting $\widetilde{\Phi}:=\left.\Phi\right|_{S}$, we obtain by (11)

$$
\max _{t \in[0,1]} \widetilde{\Phi}\left(\gamma_{i}(t)\right)<\lambda_{2}, \quad \text { for } i=1,2,3 .
$$

We recall that any component of $\mathcal{O}(r):=\{u \in S: \widetilde{\Phi}(u)<r\}$ contains at least one critical point of $\widetilde{\Phi}$, where $r>0$ [Cuesta et al. 1999, Lemma 3.6]. Note that $\mathcal{O}\left(\lambda_{2}\right)$ contains just two critical points $\varphi_{1}$ and $-\varphi_{1}$ because a critical value $c$ of $\widetilde{\Phi}$ corresponds to the eigenvalue $c$ of the negative $p$-Laplacian. Since any component of $O\left(\lambda_{2}\right)$ is path connected [ibid., Lemma 3.5], there exists a path $\gamma_{4}$ joining from $u_{-} /\left\|u_{-}\right\|_{p}$ to $\varphi_{1}$ or $-\varphi_{1}$ in $\mathcal{O}\left(\lambda_{2}\right)$. Thus, by noting that $\Phi$ is even, we can construct a path $\gamma_{0} \in \Sigma$ such that $\max _{t} \widetilde{\Phi}\left(\gamma_{0}(t)\right)<\lambda_{2}$ by considering $\gamma_{4}^{-1} \cdot \gamma_{2} \cdot \gamma_{1} \cdot \gamma_{3} \cdot\left(-\gamma_{4}\right)$ or its inverse, where $\gamma_{i}^{-1}(t):=\gamma_{i}(1-t)$ and $\gamma_{i} \cdot \gamma_{j}$ denotes the path defined by $\gamma_{i}(2 t)$ if $0 \leq t \leq \frac{1}{2}$ and $\gamma_{j}(2 t-1)$ if $\frac{1}{2}<t \leq 1$. 
3.1. Asymptotically homogeneous case near zero. We now consider the case where $A$ is asymptotically $(p-1)$-homogeneous near zero in the following sense.

(AH0) There exist a positive function $a_{0} \in C^{1}(\bar{\Omega}, \mathbb{R})$ and a continuous function $\tilde{a}_{0}(x, t)$ on $\bar{\Omega} \times[0,+\infty)$ such that

$$
A(x, y)=a_{0}(x)|y|^{p-2} y+\tilde{a}_{0}(x,|y|) y \quad \text { for every } x \in \Omega, y \in \mathbb{R}^{N},
$$

where

$$
\lim _{t \rightarrow+0} \frac{\tilde{a}_{0}(x, t)}{t^{p-2}}=0 \quad \text { uniformly in } x \in \bar{\Omega} .
$$

For this weight function $a_{0}$, we define

$$
\lambda_{1}\left(a_{0}\right):=\inf \left\{\int_{\Omega} a_{0}(x)|\nabla u|^{p} d x:\|u\|_{p}=1\right\} .
$$

Because $0<\min _{x \in \bar{\Omega}} a_{0}(x) \leq \max _{x \in \bar{\Omega}} a_{0}(x)<\infty$, by the same argument as the one for the first eigenvalue of the negative $p$-Laplacian, we can prove that $\lambda_{1}\left(a_{0}\right)$ is the first eigenvalue of

$$
-\operatorname{div}\left(a_{0}(x)|\nabla u|^{p-2} \nabla u\right)=\lambda|u|^{p-2} u \quad \text { in } \Omega, \quad u=0 \quad \text { on } \partial \Omega .
$$

Moreover, $\lambda_{1}\left(a_{0}\right)$ has a positive eigenfunction $\varphi_{a_{0}} \in C^{1}(\bar{\Omega})$ and it is simple. It is proved that (13) has no constant sign solutions other than 0 provided $\lambda \neq \lambda_{1}\left(a_{0}\right)$.

Theorem 12. Assume (AH0). For every $\varepsilon>0$ there exists $r_{0}>0$ such that equation $(\mathrm{EV} ; \lambda)$ has no nontrivial solutions in $B_{p}\left(r_{0}\right):=\left\{v \in W_{0}^{1, p}(\Omega):\|v\|_{p}<r_{0}\right\}$ provided $\lambda<\lambda_{1}\left(a_{0}\right)-\varepsilon$.

Proof. We argue by contradiction. Thus we assume that there exist $\varepsilon_{0}>0,\left\{\lambda_{n}\right\}$ and $\left\{u_{n}\right\}$ such that $\lambda_{n}<\lambda_{1}\left(a_{0}\right)-\varepsilon_{0}, u_{n} \in B_{p}(1 / n)$ and $u_{n}$ is a nontrivial solution of $\left(\mathrm{EV} ; \lambda_{n}\right)$. By taking $u_{n}$ as a test function in $\left(\mathrm{EV} ; \lambda_{n}\right)$, we have

$$
\frac{C_{0}}{p-1}\left\|\nabla u_{n}\right\|_{p}^{p} \leq \int_{\Omega} A\left(x, \nabla u_{n}\right) \nabla u_{n} d x=\lambda_{n}\left\|u_{n}\right\|_{p}^{p} \leq\left(\lambda_{1}\left(a_{0}\right)-\varepsilon_{0}\right) / n^{p} \rightarrow 0
$$

as $n \rightarrow \infty$. Therefore, $u_{n} \rightarrow 0$ in $W_{0}^{1, p}(\Omega)$. In addition, by noting that $u_{n}$ is a nontrivial solution of $\left(\mathrm{EV} ; \lambda_{n}\right)$ and $0 \leq \lambda_{n}<\lambda_{1}\left(a_{0}\right)-\varepsilon_{0}$, Proposition 4 yields that $u_{n}$ converges to 0 in $C^{1}(\bar{\Omega})$.

Set $v_{n}:=u_{n} /\left\|u_{n}\right\|_{p}$. Then it follows from (14) and the boundedness of $\left\{\lambda_{n}\right\}$ that $\left\{v_{n}\right\}$ is bounded in $W_{0}^{1, p}(\Omega)$. Hence, by choosing a subsequence, we may assume that $v_{n}$ converges to some $v_{0}$ weakly in $W_{0}^{1, p}(\Omega)$ and strongly in $L^{p}(\Omega)$. Again by taking $u_{n} /\left\|u_{n}\right\|_{p}^{p}$ as a test function in $\left(\mathrm{EV} ; \lambda_{n}\right)$, we obtain 


$$
\begin{aligned}
\lambda_{1}\left(a_{0}\right)-\varepsilon_{0}>\lambda_{n} & =\int_{\Omega} \frac{a_{0}(x)\left|\nabla u_{n}\right|^{p}}{\left\|u_{n}\right\|_{p}^{p}} d x+\int_{\Omega} \frac{\tilde{a}_{0}\left(x,\left|\nabla u_{n}\right|\right)\left|\nabla u_{n}\right|^{2}}{\left\|u_{n}\right\|_{p}^{p}} d x \\
& =\int_{\Omega} a_{0}(x)\left|\nabla v_{n}\right|^{p} d x+\int_{\Omega} \frac{\tilde{a}_{0}\left(x,\left|\nabla u_{n}\right|\right)\left|\nabla u_{n}\right|^{2}}{\left\|u_{n}\right\|_{p}^{p}} \\
& \geq \lambda_{1}\left(a_{0}\right)+\int_{\Omega} \frac{\tilde{a}_{0}\left(x,\left|\nabla u_{n}\right|\right)\left|\nabla u_{n}\right|^{2}}{\left\|u_{n}\right\|_{p}^{p}}=: \lambda_{1}\left(a_{0}\right)+I
\end{aligned}
$$

because of the characterization of $\lambda_{1}\left(a_{0}\right)$. Hypothesis (AH0) guarantees that for every $\delta>0$ there exists $\rho_{0}>0$ such that $\left|\tilde{a}_{0}(x, t)\right| \leq \delta|t|^{p-2}$ if $|t| \leq \rho_{0}$. Since $\left\|u_{n}\right\|_{C^{1}(\bar{\Omega})} \rightarrow 0$ and in view of (14), we can get

$$
|I| \leq \delta \int_{\Omega}\left|\nabla v_{n}\right|^{p} d x \leq \frac{\delta(p-1)}{C_{0}} \lambda_{n} \leq \frac{\delta(p-1)}{C_{0}}\left(\lambda_{1}\left(a_{0}\right)-\varepsilon_{0}\right)
$$

for sufficiently large $n$. As a result, by taking a sufficiently small $\delta>0$, we have a contradiction for sufficiently large $n$.

Theorem 13. Assume (AH0). For every $\varepsilon>0$ there exists $r_{1}>0$ such that $(\mathrm{EV} ; \lambda)$ has no constant sign solutions in $B_{p}\left(r_{1}\right) \backslash\{0\}$ provided $\lambda>\lambda_{1}\left(a_{0}\right)+\varepsilon$.

Proof. By way of contradiction, we assume that there exist $\varepsilon_{0}>0,\left\{\lambda_{n}\right\}$ and $\left\{u_{n}\right\}$ such that $\lambda_{n}>\lambda_{1}\left(a_{0}\right)+\varepsilon_{0}, 0 \neq u_{n} \in B_{p}(1 / n)$ and $u_{n}$ is a constant sign solution of $\left(\mathrm{EV} ; \lambda_{n}\right)$. Because $A$ is odd, we may suppose that $u_{n} \geq 0$ by considering $-u_{n}$ if necessary. Thus, by Remark 3(i)-(ii), $u_{n} \in C^{1}(\bar{\Omega})$ and $u_{n}>0$ in $\Omega$. We note that $\lambda_{n} \leq A_{p} \lambda_{1}\left(-\Delta_{p}\right)$ by Proposition 10, where $\lambda_{1}\left(-\Delta_{p}\right)$ denotes the first eigenvalue of $-\Delta_{p}$ (see (10) for the definition of $A_{p}$ ), and so $\left\{\lambda_{n}\right\}$ is bounded. Therefore, we may assume that $\lambda_{n}$ converges to some $\lambda_{0}$ by choosing a subsequence. In addition, by the same argument as in Theorem 12, we can show that $u_{n} \rightarrow 0$ in $C^{1}(\bar{\Omega})$.

Set $A_{n}(x, y):=A\left(x,\left\|u_{n}\right\|_{p} y\right) /\left\|u_{n}\right\|_{p}^{p-1}$ and $f_{n}(x, t):=\lambda_{n}|t|^{p-2} t$. Then $A_{n}$ satisfies Assumption A(i)-(iv) with the same constants $C_{0}, C_{1}$, and $C_{2}$. Moreover, $\left|f_{n}(x, t)\right| \leq \lambda_{n}|t|^{p-1} \leq A_{p} \lambda_{1}\left(-\Delta_{p}\right)|t|^{p-1}$ for every $t \in \mathbb{R}$, a.e. $x \in \Omega$. Note also that we have the boundedness of $\left\|v_{n}\right\|$ due to the inequality $C_{0}\left\|\nabla u_{n}\right\|_{p}^{p} /(p-1) \leq$ $\int_{\Omega} A\left(x, \nabla u_{n}\right) \nabla u_{n} d x=\lambda_{n}\left\|u_{n}\right\|_{p}^{p}$. Since $v_{n}:=u_{n} /\left\|u_{n}\right\|_{p}$ is a positive solution of

$$
-\operatorname{div}\left(A_{n}(x, \nabla u)\right)=f_{n}(x, u) \quad \text { in } \Omega, \quad u=0 \quad \text { on } \partial \Omega,
$$

Proposition 4 guarantees that $\left\{v_{n}\right\}$ has a convergent subsequence in $C^{1}(\bar{\Omega})$. By choosing a subsequence, we may suppose that $v_{n} \rightarrow v_{0} \neq 0$ in $C^{1}(\bar{\Omega})$ (note that $\left.\left\|v_{0}\right\|_{p}=1\right)$. Using that we obtain, for every $w \in W_{0}^{1, p}(\Omega)$, that

$$
\int_{\Omega} \frac{\tilde{a}_{0}\left(x,\left|\nabla u_{n}\right|\right) \nabla u_{n}}{\left\|u_{n}\right\|_{p}^{p-1}} \nabla w d x=\int_{\Omega} \frac{\tilde{a}_{0}\left(x,\left|\nabla u_{n}\right|\right) \nabla u_{n}}{\left|\nabla u_{n}\right|^{p-1}} \nabla w\left|\nabla v_{n}\right|^{p-1} d x \rightarrow 0
$$

as $n \rightarrow \infty$ in view of (AH0) and the convergence $u_{n} \rightarrow 0$. As a result, letting 
$n \rightarrow \infty$ in the equality

$\int_{\Omega} a_{0}(x)\left|\nabla v_{n}\right|^{p-2} \nabla v_{n} \nabla w d x+\int_{\Omega} \frac{\tilde{a}_{0}\left(x,\left|\nabla u_{n}\right|\right) \nabla u_{n}}{\left\|u_{n}\right\|_{p}^{p-1}} \nabla w d x=\lambda_{n} \int_{\Omega}\left|v_{n}\right|^{p-2} v_{n} w d x$ for each $w \in W_{0}^{1, p}(\Omega)$, we see that $v_{0} \neq 0$ is a positive solution of (13) with $\lambda=\lambda_{0}$ (see Remark 3(ii) for $v_{0}>0$ ). This yields that $\lambda_{0}=\lambda_{1}\left(a_{0}\right)$, because (13) has no positive solutions other that $\lambda=\lambda_{1}\left(a_{0}\right)$. Therefore we have a contradiction, because $\lambda_{0}=\lim _{n \rightarrow \infty} \lambda_{n} \geq \lambda_{1}\left(a_{0}\right)+\varepsilon_{0}$.

Proposition 14. Assume (AH0). Then, for every $\varepsilon>0$, there exists $r_{0}>0$ such that

$$
\frac{\underline{\lambda}_{1}(A, r)}{r^{p}} \geq \lambda_{1}\left(a_{0}\right)-\varepsilon \quad \text { for every } 0<r<r_{0} .
$$

Proof. Assume that there exist $\varepsilon>0$ and $r_{n}>0$ such that $r_{n} \rightarrow 0$ as $n \rightarrow \infty$ and $\underline{\lambda}_{1}\left(A, r_{n}\right) / r_{n}^{p}<\lambda_{1}\left(a_{0}\right)-\varepsilon$ for every $n \in \mathbb{N}$. Because of Proposition 5 and Lemma 6 (note that $A$ is odd in the second variable), we can choose a positive function $u_{n} \in\left(r_{n} S\right) \cap C^{1}(\bar{\Omega})$ satisfying

$$
\int_{\Omega} A\left(x, \nabla u_{n}\right) \nabla u_{n} d x=\underline{\lambda}_{1}\left(A, r_{n}\right), \quad \min _{v \in r_{n} S} \int_{\Omega} G(x, \nabla v) d x=\int_{\Omega} G\left(x, \nabla u_{n}\right) d x .
$$

Note that

$$
\frac{C_{0}}{p-1}\left\|\nabla u_{n}\right\|_{p}^{p} \leq \int_{\Omega} A\left(x, \nabla u_{n}\right) \nabla u_{n} d x=\underline{\lambda}_{1}\left(A, r_{n}\right)<\left(\lambda_{1}\left(a_{0}\right)-\varepsilon\right) r_{n}^{p} \rightarrow 0,
$$

and so $u_{n} \rightarrow 0$ in $W_{0}^{1, p}(\Omega)$. Because $u_{n}$ is a solution of $(\mathrm{EV} ; \lambda)$ with $\lambda=$ $\underline{\lambda}_{1}\left(A, r_{n}\right) / r_{n}^{p}$ (see Proposition 5), by combining the inequality

$$
\lambda_{1}\left(a_{0}\right)-\varepsilon>\frac{\underline{\lambda}_{1}\left(A, r_{n}\right)}{r_{n}^{p}}=\int_{\Omega} a_{0}(x)\left|\nabla v_{n}\right|^{p} d x+\int_{\Omega} \frac{\tilde{a}_{0}\left(x,\left|\nabla u_{n}\right|\right)\left|\nabla u_{n}\right|^{2}}{\left\|u_{n}\right\|_{p}^{p}} d x
$$

and an argument as in Theorem 12 with $\lambda_{n}=\underline{\lambda}_{1}\left(A, r_{n}\right) / r_{n}^{p}$, we have a contradiction.

Proposition 15. Assume (AH0). Then, for every $\varepsilon>0$, there exists $r_{1}>0$ such that

$$
\frac{\bar{\lambda}_{1}(A, r)}{r^{p}} \leq \lambda_{1}\left(a_{0}\right)+\varepsilon \quad \text { for every } 0<r<r_{1} .
$$

Proof. Assume that there exist $\varepsilon_{0}>0$ and $r_{n}>0$ such that $r_{n} \rightarrow 0$ as $n \rightarrow \infty$ and $\bar{\lambda}_{1}\left(A, r_{n}\right) / r_{n}^{p}>\lambda_{1}\left(a_{0}\right)+\varepsilon_{0}$ for every $n \in \mathbb{N}$. According to Lemma 6 and Proposition 5, we can take a positive function $u_{n} \in\left(r_{n} S\right) \cap C^{1}(\bar{\Omega})$ satisfying

$$
\int_{\Omega} A\left(x, \nabla u_{n}\right) \nabla u_{n} d x=\bar{\lambda}_{1}\left(A, r_{n}\right), \quad \min _{v \in r_{n} S} \int_{\Omega} G(x, \nabla v) d x=\int_{\Omega} G\left(x, \nabla u_{n}\right) d x .
$$

Noting that, with $\varphi_{a_{0}}$ the positive eigenfunction corresponding to $\lambda_{1}\left(a_{0}\right)$ satisfying 
$\left\|\varphi_{a_{0}}\right\|_{p}=1$, we have

$\frac{C_{0}}{p(p-1)}\left\|\nabla u_{n}\right\|_{p}^{p} \leq \int_{\Omega} G\left(x, \nabla u_{n}\right) d x \leq \int_{\Omega} G\left(x, r_{n} \nabla \varphi_{a_{0}}\right) d x \leq \frac{C_{1} r_{n}^{p}}{p(p-1)}\left\|\nabla \varphi_{a_{0}}\right\|_{p}^{p}$,

we see that $u_{n} \rightarrow 0$ in $C^{1}(\bar{\Omega})$ due to Proposition 4 , because $u_{n}$ is a positive solution of $(\mathrm{EV} ; \lambda)$ with $\lambda=\bar{\lambda}_{1}\left(A, r_{n}\right) / r_{n}^{p}$ and $\left(\lambda_{1}\left(a_{0}\right)+\varepsilon_{0}<\right) \bar{\lambda}_{1}\left(A, r_{n}\right) / r_{n}^{p} \leq A_{p} \lambda_{1}\left(-\Delta_{p}\right)$ by Proposition 10, where $\lambda_{1}\left(-\Delta_{p}\right)$ denotes the first eigenvalue of $-\Delta_{p}$ (see (10) for the definition of $A_{p}$ ). Therefore, by the same argument as in Theorem 13 with $\lambda_{n}=\bar{\lambda}_{1}\left(A, r_{n}\right) / r_{n}^{p}$, we have a contradiction.

The following result follows from Propositions 14 and 15 , (note $\underline{\lambda}_{1}(A, r) \leq$ $\bar{\lambda}_{1}(A, r)$ for every $\left.r>0\right)$.

Corollary 16. Under (AHO), we have

$$
\lim _{r \rightarrow+0} \frac{\bar{\lambda}_{1}(A, r)}{r^{p}}=\lim _{r \rightarrow+0} \frac{\underline{\lambda}_{1}(A, r)}{r^{p}}=\lambda_{1}\left(a_{0}\right) .
$$

Proposition 17. Under (AH0), we have

$$
\lim _{r \rightarrow+0} \frac{\mu_{1}(A, r)}{r^{p}}=\frac{\lambda_{1}\left(a_{0}\right)}{p} .
$$

Proof. Due to Proposition 5, for every $r>0$, there exists a positive solution $u_{r} \in(r S) \cap C^{1}(\bar{\Omega})$ of $(\mathrm{EV} ; \lambda)$ with $\lambda=\lambda_{1}\left(A, u_{r}\right) / r^{p}$ and $\mu_{1}(A, r)=J\left(u_{r}\right)$. Then we can prove that $u_{r} \rightarrow 0$ in $C^{1}(\bar{\Omega})$ as $r \rightarrow+0$ and $u_{r} /\left\|u_{r}\right\|_{p}$ is bounded in $W_{0}^{1, p}(\Omega)$ as $r \rightarrow+0$ by a similar reason to the one in Proposition 15 (note that $\lambda_{1}\left(A, u_{r}\right) / r^{p}$ is bounded as $r \rightarrow+0$ by the inequality below and Corollary 16).

Set $\widetilde{G}_{0}(x, y):=\int_{0}^{|y|} \tilde{a}_{0}(x, t) t d t$ for $y \in \mathbb{R}^{N}$. We point out that

$$
\underline{\lambda}_{1}(A, r) \leq \lambda_{1}\left(A, u_{r}\right) \leq \bar{\lambda}_{1}(A, r)
$$

and

$$
\begin{aligned}
\mu_{1}(A, r) & =\int_{\Omega} G\left(x, \nabla u_{r}\right) d x=\frac{1}{p} \int_{\Omega} a_{0}(x)\left|\nabla u_{r}\right|^{p} d x+\int_{\Omega} \widetilde{G}_{0}\left(x, \nabla u_{r}\right) d x \\
& =\frac{\lambda_{1}\left(A, u_{r}\right)}{p}-\frac{1}{p} \int_{\Omega} \tilde{a}_{0}(x,|\nabla u|)\left|\nabla u_{r}\right|^{2} d x+\int_{\Omega} \widetilde{G}_{0}\left(x, \nabla u_{r}\right) d x .
\end{aligned}
$$

Thus, by Corollary 16 and $r=\left\|u_{r}\right\|_{p}$, it suffices to prove

$$
\lim _{r \rightarrow+0} \int_{\Omega} \frac{\tilde{a}_{0}(x,|\nabla u|)\left|\nabla u_{r}\right|^{2}}{\left\|u_{r}\right\|_{p}^{p}} d x=0 \quad \text { and } \quad \lim _{r \rightarrow+0} \int_{\Omega} \frac{\widetilde{G}_{0}\left(x, \nabla u_{r}\right)}{\left\|u_{r}\right\|_{p}^{p}} d x=0 .
$$

Now we fix any $\varepsilon>0$. Then, by (AH0), there exists $\delta>0$ such that

$$
\left|\tilde{a}_{0}(x, t)\right| \leq \varepsilon t^{p-2} \quad \text { and } \quad\left|\widetilde{G}_{0}(x, y)\right| \leq \varepsilon|y|^{p} / p \quad \text { for every } 0<t \leq \delta,|y| \leq \delta .
$$


Because $u_{r} \rightarrow 0$ in $C^{1}(\bar{\Omega})$ as $r \rightarrow+0$, we may assume that $\left\|u_{r}\right\|_{C^{1}(\bar{\Omega})} \leq \delta$ for sufficiently small $r>0$. Therefore, we obtain

$$
\left|\int_{\Omega} \frac{\tilde{a}_{0}(x,|\nabla u|)\left|\nabla u_{r}\right|^{2}}{\left\|u_{r}\right\|_{p}^{p}} d x\right| \leq \varepsilon \frac{\left\|\nabla u_{r}\right\|_{p}^{p}}{\left\|u_{r}\right\|_{p}^{p}}, \quad\left|\int_{\Omega} \frac{\widetilde{G}_{0}\left(x, \nabla u_{r}\right)}{\left\|u_{r}\right\|_{p}^{p}} d x\right| \leq \varepsilon \frac{\left\|\nabla u_{r}\right\|_{p}^{p}}{p\left\|u_{r}\right\|_{p}^{p}} .
$$

Since $\left\|\nabla u_{r}\right\|_{p} /\left\|u_{r}\right\|_{p}$ is bounded as $r \rightarrow+0$ and $\varepsilon>0$ is arbitrary, our conclusion holds.

3.2. Asymptotically homogeneous case near $\infty$. In this subsection, we consider the case where $A$ is asymptotically ( $p-1)$-homogeneous near $\infty$ in the following sense.

(AH) There exist a positive function $a_{\infty} \in C^{1}(\bar{\Omega}, \mathbb{R})$ and a continuous function $\tilde{a}(x, t)$ on $\bar{\Omega} \times \mathbb{R}$ such that

$$
A(x, y)=a_{\infty}(x)|y|^{p-2} y+\tilde{a}(x,|y|) y \quad \text { for every } x \in \Omega, y \in \mathbb{R}^{N},
$$

where

$$
\lim _{t \rightarrow+\infty} \frac{\tilde{a}(x, t)}{t^{p-2}}=0 \quad \text { uniformly in } x \in \bar{\Omega} .
$$

For the weight function $a_{\infty}$, we define

$$
\lambda_{1}\left(a_{\infty}\right):=\inf \left\{\int_{\Omega} a_{\infty}(x)|\nabla u|^{p} d x:\|u\|_{p}=1\right\} .
$$

Because $0<\min _{x \in \bar{\Omega}} a_{\infty}(x) \leq \max _{x \in \bar{\Omega}} a_{\infty}(x)<\infty$, by the same argument as for the first eigenvalue of $-\Delta_{p}$, we can prove the following elementary results:

(i) $\lambda_{1}\left(a_{\infty}\right)$ is the first eigenvalue of

$$
-\operatorname{div}\left(a_{\infty}(x)|\nabla u|^{p-2} \nabla u\right)=\lambda|u|^{p-2} u \quad \text { in } \Omega, \quad u=0 \quad \text { on } \partial \Omega .
$$

(ii) $\lambda_{1}\left(a_{\infty}\right)$ has a positive eigenfunction $\varphi_{a_{\infty}} \in C^{1}(\bar{\Omega})$ with $\left\|\varphi_{a_{\infty}}\right\|_{p}=1$ and it is simple.

(iii) If $\lambda \neq \lambda_{1}\left(a_{\infty}\right)$, then (17) has no constant sign solutions other than 0 .

Theorem 18. Assume (AH). For every $\varepsilon>0$ there exists $R_{0}>0$ such that equation $(\mathrm{EV} ; \lambda)$ has no solutions in $W_{0}^{1, p}(\Omega) \backslash B_{p}\left(R_{0}\right)$ provided $\lambda<\lambda_{1}\left(a_{\infty}\right)-\varepsilon$.

To prove the theorem, we need the following result.

Lemma 19. Assume $(\mathrm{AH})$ and let $\left\{u_{n}\right\} \subset W_{0}^{1, p}(\Omega)$ be a sequence satisfying $\left\|u_{n}\right\|_{p} \rightarrow \infty$ as $n \rightarrow \infty$. If $v_{n}:=u_{n} /\left\|u_{n}\right\|_{p}$ is bounded in $W_{0}^{1, p}(\Omega)$, the following assertions hold:

(i) $\lim _{n \rightarrow \infty} \int_{\Omega} \frac{\tilde{a}\left(x,\left|\nabla u_{n}\right|\right)\left|\nabla u_{n}\right|^{2}}{\left\|u_{n}\right\|_{p}^{p}} d x=0$. 
(ii) For every $w \in W_{0}^{1, p}(\Omega)$,

$$
\lim _{n \rightarrow \infty} \int_{\Omega} \frac{\tilde{a}\left(x,\left|\nabla u_{n}\right|\right) \nabla u_{n} \nabla w}{\left\|u_{n}\right\|_{p}^{p-1}} d x=0 .
$$

(iii) $\lim _{n \rightarrow \infty} \int_{\Omega} \frac{\widetilde{G}\left(x, \nabla u_{n}\right)}{\left\|u_{n}\right\|_{p}^{p}} d x=0$, where $\widetilde{G}(x, y):=\int_{0}^{|y|} \tilde{a}(x, t) t d t \quad$ for $y \in \mathbb{R}^{N}$. Proof. (i) Fix any $\varepsilon>0$. By the property of the function $\tilde{a}$, there exist $R>0$ and $C>0$ such that

$$
|\tilde{a}(x, t)| \leq \varepsilon|t|^{p-2} \text { if } t \geq R \quad \text { and } \quad|\tilde{a}(x, t)| \leq C \text { if } 0 \leq t \leq R .
$$

Therefore, we obtain

$$
\begin{aligned}
\left|\int_{\Omega} \frac{\tilde{a}\left(x,\left|\nabla u_{n}\right|\right)\left|\nabla u_{n}\right|^{2}}{\left\|u_{n}\right\|_{p}^{p}} d x\right| & \leq \int_{\left|\nabla u_{n}\right|>R} \varepsilon\left|\nabla v_{n}\right|^{p} d x+\int_{\left|\nabla u_{n}\right| \leq R} \frac{C\left|\nabla u_{n}\right|^{2}}{\left\|u_{n}\right\|_{p}^{p}} d x \\
& \leq \varepsilon\left\|\nabla v_{n}\right\|_{p}^{p}+\frac{C R^{2}|\Omega|}{\left\|u_{n}\right\|_{p}^{p}} \leq \varepsilon D^{p}+\frac{C R^{2}|\Omega|}{\left\|u_{n}\right\|_{p}^{p}}
\end{aligned}
$$

by (18), where $D:=\sup _{n}\left\|\nabla v_{n}\right\|_{p}$. Letting $n \rightarrow \infty$, we have

$$
\limsup _{n \rightarrow \infty}\left|\int_{\Omega} \frac{\tilde{a}\left(x,\left|\nabla u_{n}\right|\right)\left|\nabla u_{n}\right|^{2}}{\left\|u_{n}\right\|_{p}^{p}} d x\right| \leq \varepsilon D^{p}
$$

because $\left\|u_{n}\right\|_{p} \rightarrow \infty$ as $n \rightarrow \infty$. Thus, since $\varepsilon>0$ is arbitrary, our conclusion holds.

(ii) For any $\varepsilon>0$ and $w \in W_{0}^{1, p}(\Omega)$, we have

$$
\begin{aligned}
\mid \int_{\Omega} \frac{\tilde{a}\left(x,\left|\nabla u_{n}\right|\right) \nabla u_{n} \nabla w}{\left\|u_{n}\right\|_{p}^{p-1}} & d x \mid \\
& \leq \int_{\left|\nabla u_{n}\right|>R} \varepsilon\left|\nabla v_{n}\right|^{p-1}|\nabla w| d x+\int_{\left|\nabla u_{n}\right| \leq R} \frac{C\left|\nabla u_{n}\right||\nabla w|}{\left\|u_{n}\right\|_{p}^{p-1}} d x \\
& \leq \varepsilon\left\|\nabla v_{n}\right\|_{p}^{p-1}\|\nabla w\|_{p}+\frac{C R\|\nabla w\|_{p}|\Omega|^{(p-1) / p}}{\left\|u_{n}\right\|_{p}^{p-1}}
\end{aligned}
$$

by Hölder's inequality and (18). By combining this inequality and a similar argument to that used in (i), our conclusion is shown.

(iii) It is easily shown that, for every $\varepsilon>0$, there exists $C>0$ such that

$$
|\widetilde{G}(x, y)| \leq \varepsilon|y|^{p}+C \text { for every } y \in \mathbb{R}^{N} \text {. }
$$

Therefore, $\left|\int_{\Omega} \widetilde{G}\left(x, \nabla u_{n}\right) d x\right| \leq \varepsilon\left\|\nabla u_{n}\right\|_{p}^{p}+C|\Omega|$. This implies our conclusion. 
Proof of Theorem 18. By way of contradiction, we assume that there exist $\varepsilon_{0}>0$, $\left\{\lambda_{n}\right\}$, and $\left\{u_{n}\right\}$ such that $\lambda_{n}<\lambda_{1}\left(a_{\infty}\right)-\varepsilon_{0}, \lim _{n \rightarrow \infty}\left\|u_{n}\right\|_{p}=\infty$, and $u_{n}$ is a solution of $\left(\mathrm{EV} ; \lambda_{n}\right)$. By taking $u_{n}$ as a test function in $\left(\mathrm{EV} ; \lambda_{n}\right)$, we have

$$
\frac{C_{0}}{p-1}\left\|\nabla u_{n}\right\|_{p}^{p} \leq \int_{\Omega} A\left(x, \nabla u_{n}\right) \nabla u_{n} d x=\lambda_{n}\left\|u_{n}\right\|_{p}^{p} \leq\left(\lambda_{1}\left(a_{\infty}\right)-\varepsilon_{0}\right)\left\|u_{n}\right\|_{p}^{p} ;
$$

refer to Remark 1(iii). Therefore, $v_{n}:=u_{n} /\left\|u_{n}\right\|_{p}$ is bounded in $W_{0}^{1, p}(\Omega)$.

Again by taking $u_{n} /\left\|u_{n}\right\|_{p}^{p}$ as a test function in $\left(\mathrm{EV} ; \lambda_{n}\right)$, we obtain

$$
\begin{aligned}
\lambda_{1}\left(a_{\infty}\right)-\varepsilon_{0}>\lambda_{n} & =\int_{\Omega} \frac{a_{\infty}(x)\left|\nabla u_{n}\right|^{p}}{\left\|u_{n}\right\|_{p}^{p}} d x+\int_{\Omega} \frac{\tilde{a}\left(x,\left|\nabla u_{n}\right|\right)\left|\nabla u_{n}\right|^{2}}{\left\|u_{n}\right\|_{p}^{p}} d x \\
& =\int_{\Omega} a_{\infty}(x)\left|\nabla v_{n}\right|^{p} d x+\int_{\Omega} \frac{\tilde{a}\left(x,\left|\nabla u_{n}\right|\right)\left|\nabla u_{n}\right|^{2}}{\left\|u_{n}\right\|_{p}^{p}} d x \\
& \geq \lambda_{1}\left(a_{\infty}\right)+o(1),
\end{aligned}
$$

using the definition of $\lambda_{1}\left(a_{\infty}\right)$ and Lemma 19(i). This is a contradiction.

Theorem 20. Assume (AH). For every $\varepsilon>0$ there exists $R_{1}>0$ such that $(\mathrm{EV} ; \lambda)$ has no constant sign solutions in $W_{0}^{1, p}(\Omega) \backslash B_{p}\left(R_{1}\right)$ provided $\lambda>\lambda_{1}\left(a_{\infty}\right)+\varepsilon$.

Proof. By way of contradiction, we assume that there exist $\varepsilon_{0}>0,\left\{\lambda_{n}\right\}$, and $\left\{u_{n}\right\}$ such that $\lambda_{n}>\lambda_{1}\left(a_{\infty}\right)+\varepsilon_{0}, \lim _{n \rightarrow \infty}\left\|u_{n}\right\|_{p}=\infty$, and $u_{n}$ is a constant sign solution of $\left(\mathrm{EV} ; \lambda_{n}\right)$. Because $A$ is odd, we may suppose that $u_{n} \geq 0$ by considering $-u_{n}$ if necessary. Thus, by Remark $3, u_{n} \in C^{1}(\bar{\Omega})$ and $u_{n}>0$ in $\Omega$. Here we note that $\lambda_{n} \leq A_{p} \lambda_{1}\left(-\Delta_{p}\right)$ by Proposition 10, where $\lambda_{1}\left(-\Delta_{p}\right)$ denotes the first eigenvalue of $-\Delta_{p}$ (see (10) for the definition of $A_{p}$ ), and so $\left\{\lambda_{n}\right\}$ is bounded. Hence we may assume, by taking a subsequence, that $\lambda_{n}$ converges to some

$$
\lambda_{0} \in\left[\lambda_{1}\left(a_{\infty}\right)+\varepsilon_{0}, A_{p} \lambda_{1}\left(-\Delta_{p}\right)\right] .
$$

In addition, we know that $v_{n}:=u_{n} /\left\|u_{n}\right\|_{p}$ is bounded in $W_{0}^{1, p}(\Omega)$

$$
\frac{C_{0}}{p-1}\left\|\nabla u_{n}\right\|_{p}^{p} \leq \int_{\Omega} A\left(x, \nabla u_{n}\right) d x=\lambda_{n}\left\|u_{n}\right\|_{p}^{p},
$$

where we take $u_{n}$ as a test function in $\left(\mathrm{EV} ; \lambda_{n}\right)$. Thus, by choosing a subsequence, we may suppose that $v_{n}$ converges to some $v$ weakly in $W_{0}^{1, p}(\Omega)$ and strongly in $L^{p}(\Omega)$.

We claim that $v$ is a positive solution of

$$
-\operatorname{div}\left(a_{\infty}(x)|\nabla v|^{p-2} \nabla v\right)=\lambda_{0}|v|^{p-2} v \quad \text { in } \Omega, \quad v=0 \quad \text { on } \partial \Omega,
$$

that is, $v$ is a positive eigenfunction corresponding to $\lambda_{0}$. If our claim holds, then $\lambda_{0}=\lambda_{1}\left(a_{\infty}\right)$ occurs because (17) has no positive solutions in the case of $\lambda \neq \lambda_{1}\left(a_{\infty}\right)$. Hence this contradicts $\lambda_{1}\left(a_{\infty}\right)+\varepsilon_{0} \leq \lim _{n \rightarrow \infty} \lambda_{n}=\lambda_{0}$. 
We now prove our claim. First, we show that $v_{n}$ converges to $v$ strongly in $W_{0}^{1, p}(\Omega)$. Indeed, by taking $\left(v_{n}-v\right) /\left\|u_{n}\right\|_{p}^{p-1}$ as a test function in $\left(\mathrm{EV} ; \lambda_{n}\right)$, we have

$$
\begin{aligned}
\lambda_{n} \int_{\Omega} & v_{n}^{p-1}\left(v_{n}-v\right) d x \\
& =\int_{\Omega} a_{\infty}(x)\left|\nabla v_{n}\right|^{p-2} \nabla v_{n} \nabla\left(v_{n}-v\right) d x+\int_{\Omega} \frac{\tilde{a}\left(x,\left|\nabla u_{n}\right|\right) \nabla u_{n}}{\left\|u_{n}\right\|_{p}^{p-1}} \nabla\left(v_{n}-v\right) d x \\
& =\int_{\Omega} a_{\infty}(x)\left|\nabla v_{n}\right|^{p-2} \nabla v_{n} \nabla\left(v_{n}-v\right) d x+o(1)
\end{aligned}
$$

as $n \rightarrow \infty$ due to Lemma 19(i)-(ii). Since $v_{n} \rightarrow v$ in $L^{p}(\Omega)$, this implies that $\int_{\Omega} a_{\infty}(x)\left|\nabla v_{n}\right|^{p-2} \nabla v_{n} \nabla\left(v_{n}-v\right) d x$ converges to 0 as $n \rightarrow \infty$. Noting that

$$
\begin{aligned}
o(1) & =\int_{\Omega} a_{\infty}(x)\left(\left|\nabla v_{n}\right|^{p-2} \nabla v_{n}-|\nabla v|^{p-2} \nabla v\right) \nabla\left(v_{n}-v\right) d x \\
& \geq \min _{\bar{\Omega}} a_{\infty} \int_{\Omega}\left(\left|\nabla v_{n}\right|^{p-2} \nabla v_{n}-|\nabla v|^{p-2} \nabla v\right) \nabla\left(v_{n}-v\right) d x \\
& \geq \min _{\bar{\Omega}} a_{\infty}\left(\left\|\nabla v_{n}\right\|_{p}^{p-1}-\|\nabla v\|_{p}^{p-1}\right)\left(\left\|\nabla v_{n}\right\|_{p}-\|\nabla v\|_{p}\right) \geq 0,
\end{aligned}
$$

we have $v_{n} \rightarrow v$ in $W_{0}^{1, p}(\Omega)$ (note $0<\min _{\bar{\Omega}} a_{\infty} \leq \max _{\bar{\Omega}} a_{\infty}<\infty$ ). As a result, $v$ is a solution of (19) by letting $n \rightarrow \infty$ in the equality

$$
\int_{\Omega} a_{\infty}(x)\left|\nabla v_{n}\right|^{p-2} \nabla v_{n} \nabla w d x+\int_{\Omega} \frac{\tilde{a}\left(x,\left|\nabla u_{n}\right|\right) \nabla u_{n} \nabla w}{\left\|u_{n}\right\|_{p}^{p-1}} d x=\lambda_{n} \int_{\Omega} v_{n}^{p-1} w d x
$$

for every $w \in W_{0}^{1, p}(\Omega)$; note that, by Lemma 19(ii), the second term converges to zero. Since $v_{n}=u_{n} /\left\|u_{n}\right\|_{p}>0$ in $\Omega, v$ is nonnegative, and so $v$ is positive by Remark 3(i) and $\|v\|_{p}=1$. Thus our claim is shown.

Proposition 21. Assume (AH). Then, for every $\varepsilon>0$, there exists $R_{0}>0$ such that

$$
\frac{\underline{\lambda}_{1}(A, r)}{r^{p}} \geq \lambda_{1}\left(a_{\infty}\right)-\varepsilon \quad \text { for every } r>R_{0} \text {. }
$$

Proof. Assume that there exist $\varepsilon_{0}>0$ and $r_{n}>0$ such that $r_{n} \rightarrow \infty$ as $n \rightarrow \infty$ and $\underline{\lambda}_{1}\left(A, r_{n}\right) / r_{n}^{p}<\lambda_{1}\left(a_{\infty}\right)-\varepsilon_{0}$ for every $n \in \mathbb{N}$. Because of Proposition 5 and Lemma 6 , we can choose a positive function $u_{n} \in\left(r_{n} S\right) \cap C^{1}(\bar{\Omega})$ satisfying

$$
\int_{\Omega} A\left(x, \nabla u_{n}\right) \nabla u_{n} d x=\underline{\lambda}_{1}\left(A, r_{n}\right), \quad \min _{v \in r_{n} S} \int_{\Omega} G(x, \nabla v) d x=\int_{\Omega} G\left(x, \nabla u_{n}\right) d x .
$$

Note that

$$
\frac{C_{0}}{p-1}\left\|\nabla u_{n}\right\|_{p}^{p} \leq \int_{\Omega} A\left(x, \nabla u_{n}\right) \nabla u_{n} d x=\underline{\lambda}_{1}\left(A, r_{n}\right)<\left(\lambda_{1}\left(a_{\infty}\right)-\varepsilon_{0}\right) r_{n}^{p},
$$


and so $u_{n} / r_{n}=u_{n} /\left\|u_{n}\right\|_{p}$ is bounded in $W_{0}^{1, p}(\Omega)$. Because $u_{n}$ is a solution of $(\mathrm{EV} ; \lambda)$ with $\lambda=\underline{\lambda}_{1}\left(A, r_{n}\right) / r_{n}^{p}$ (see Proposition 5 ), by the same argument as in Theorem 18 with $\lambda_{n}=\underline{\lambda}_{1}\left(A, r_{n}\right) / r_{n}^{p}$, we have a contradiction.

Proposition 22. Assume (AH). Then, for every $\varepsilon>0$, there exists $R_{1}>0$ such that $\frac{\bar{\lambda}_{1}(A, r)}{r^{p}} \leq \lambda_{1}\left(a_{\infty}\right)+\varepsilon \quad$ for every $r>R_{1}$.

Proof. Assume that there exist $\varepsilon_{0}>0$ and $r_{n}>0$ such that $r_{n} \rightarrow \infty$ as $n \rightarrow \infty$ and $\bar{\lambda}_{1}\left(A, r_{n}\right) / r_{n}^{p}>\lambda_{1}\left(a_{\infty}\right)+\varepsilon_{0}$ for every $n \in \mathbb{N}$. According to Lemma 6 and Proposition 5, we can take a positive function $u_{n} \in\left(r_{n} S\right) \cap C^{1}(\bar{\Omega})$ satisfying

$$
\int_{\Omega} A\left(x, \nabla u_{n}\right) \nabla u_{n} d x=\bar{\lambda}_{1}\left(A, r_{n}\right), \quad \min _{v \in r_{n} S} \int_{\Omega} G(x, \nabla v) d x=\int_{\Omega} G\left(x, \nabla u_{n}\right) d x .
$$

Note that, with $\varphi_{a_{\infty}}$ as in item (ii) of page 165 , we have

$\frac{C_{0}}{p(p-1)}\left\|\nabla u_{n}\right\|_{p}^{p} \leq \int_{\Omega} G\left(x, \nabla u_{n}\right) d x \leq \int_{\Omega} G\left(x, r_{n} \nabla \varphi_{a_{\infty}}\right) d x \leq \frac{C_{1} r_{n}^{p}}{p(p-1)}\left\|\nabla \varphi_{a_{\infty}}\right\|_{p}^{p}$.

Hence $u_{n} / r_{n}=u_{n} /\left\|u_{n}\right\|_{p}$ is bounded in $W_{0}^{1, p}(\Omega)$. Since $u_{n}$ is a positive solution of $(\mathrm{EV} ; \lambda)$ with $\lambda=\bar{\lambda}_{1}\left(A, r_{n}\right) / r_{n}^{p}$, by the same argument as in Theorem 20 with $\lambda_{n}=\bar{\lambda}_{1}\left(A, r_{n}\right) / r_{n}^{p}$, we have a contradiction.

By Propositions 21 and 22, we have the following result.

Corollary 23. Under (AH), we have

$$
\lim _{r \rightarrow+\infty} \frac{\bar{\lambda}_{1}(A, r)}{r^{p}}=\lim _{r \rightarrow+\infty} \frac{\underline{\lambda}_{1}(A, r)}{r^{p}}=\lambda_{1}\left(a_{\infty}\right) .
$$

Proposition 24. Under (AH), we have

$$
\lim _{r \rightarrow+\infty} \frac{\mu_{1}(A, r)}{r^{p}}=\frac{\lambda_{1}\left(a_{\infty}\right)}{p} .
$$

Proof. Due to Proposition 5, for every $r>0$, there exists a positive solution $u_{r} \in(r S) \cap C^{1}(\bar{\Omega})$ of $(\mathrm{EV} ; \lambda)$ with $\lambda=\lambda_{1}\left(A, u_{r}\right) / r^{p}$ and $\mu_{1}(A, r)=J\left(u_{r}\right)$. Then $u_{r} /\left\|u_{r}\right\|_{p}=u_{r} / r$ is bounded in $W_{0}^{1, p}(\Omega)$, as seen from

$$
\frac{C_{0}}{p(p-1)}\left\|\nabla u_{r}\right\|_{p}^{p} \leq \int_{\Omega} G\left(x, \nabla u_{r}\right) d x \leq \int_{\Omega} G(x, r \nabla w) d x \leq \frac{r^{p} C_{1}}{p(p-1)}\|\nabla w\|_{p}^{p}
$$

for any $w \in W_{0}^{1, p}(\Omega)$ with $\|w\|_{p}=1$.

Set

$$
\widetilde{G}(x, y):=\int_{0}^{|y|} \tilde{a}(x, t) t d x \quad \text { for } y \in \mathbb{R}^{N} .
$$

Note that

$$
\underline{\lambda}_{1}(A, r) \leq \lambda_{1}\left(A, u_{r}\right) \leq \bar{\lambda}_{1}(A, r)
$$


and

$$
\begin{aligned}
\mu_{1}(A, r) & =\int_{\Omega} G\left(x, \nabla u_{r}\right) d x=\frac{1}{p} \int_{\Omega} a_{\infty}(x)\left|\nabla u_{r}\right|^{p} d x+\int_{\Omega} \widetilde{G}\left(x, \nabla u_{r}\right) d x \\
& =\frac{\lambda_{1}\left(A, u_{r}\right)}{p}-\frac{1}{p} \int_{\Omega} \tilde{a}(x,|\nabla u|)\left|\nabla u_{r}\right|^{2} d x+\int_{\Omega} \widetilde{G}\left(x, \nabla u_{r}\right) d x .
\end{aligned}
$$

According to Corollary 23 and Lemma 19(i) and (iii) (note $\left\|u_{r}\right\|_{p}=r \rightarrow+\infty$ ), our conclusion is achieved.

\section{Existence of a positive solution}

In this section, we provide the existence of a positive solution to the equation

$$
\begin{cases}-\operatorname{div} A(x, \nabla u)=f(x, u) & \text { in } \Omega, \\ u=0 & \text { on } \partial \Omega,\end{cases}
$$

where the nonlinear term $f$ satisfies Assumption $(f)$.

Theorem 25. Assume (AH0), (AH), and $(f)$. Let $\lambda_{1}\left(a_{0}\right)$ and $\lambda_{1}\left(a_{\infty}\right)$ be the first eigenvalues of, respectively, (13) and (17) (see the discussion there). If one of the following conditions holds, $(\mathrm{P})$ has at least one positive solution.

(i) $\alpha_{0}>\lambda_{1}\left(a_{0}\right)$ and $\alpha<\lambda_{1}\left(a_{\infty}\right)$.

(ii) $\alpha_{0}<\lambda_{1}\left(a_{0}\right)$ and $\alpha>\lambda_{1}\left(a_{\infty}\right)$.

This addresses the existence of an eigenvalue for our operator because we can apply Theorem 25 to $f(x, u)=\lambda|u|^{p-2} u$.

Corollary 26. Assume (AH0), $(\mathrm{AH})$, and $\lambda_{1}\left(a_{0}\right) \neq \lambda_{1}\left(a_{\infty}\right)$. Then, for every $\lambda$ between $\lambda_{1}\left(a_{0}\right)$ and $\lambda_{1}\left(a_{\infty}\right),(\mathrm{EV} ; \lambda)$ has a nontrivial (positive) solution. Therefore $\lambda$ is an eigenvalue of $A$

To show the existence of a positive solution, we define a $C^{1}$ functional $I$ on $W_{0}^{1, p}(\Omega)$ by

$$
I(u):=\int_{\Omega} G(x, \nabla u) d x-\int_{\Omega} F_{+}(x, u) d x \quad \text { for } u \in W_{0}^{1, p}(\Omega),
$$

where $F_{+}(x, u):=\int_{0}^{u} f_{+}(x, u) d x$, with $f_{+}(x, t)$ given by $f(x, t)$ if $t \geq 0$ and 0 if $t \leq 0$.

Remark 27. If $u \in W_{0}^{1, p}(\Omega)$ is a nontrivial critical point of $I$, then $u$ is a positive solution of $(\mathrm{P})$.

Indeed, by taking $-u_{-}$as a test function, we obtain

$$
\begin{aligned}
0=\left\langle I^{\prime}(u),-u_{-}\right\rangle & =\int_{\Omega} A(x, \nabla u)\left(-\nabla u_{-}\right) d x-\int_{\Omega} f_{+}(x, u)\left(-u_{-}\right) d x \\
& =\int_{\Omega} A(x, \nabla u)\left(-\nabla u_{-}\right) d x \geq \frac{C_{0}}{p-1}\left\|\nabla u_{-}\right\|_{p}^{p} .
\end{aligned}
$$


Thus $u \geq 0$. By Remark 3(ii) (note that $u \neq \equiv 0$ ), we see that $u$ is a positive solution of $(\mathrm{P})$ (note that $f_{+}(x, u)=f(x, u)$ ).

Convention. From now on, let Assumption $(f)$ be satisfied.

Lemma 28. If $\alpha \neq \lambda_{1}\left(a_{\infty}\right)$, then I satisfies the Palais-Smale condition.

Proof. Let $\left\{u_{n}\right\}$ be a Palais-Smale sequence of $I$, which means that

$$
I\left(u_{n}\right) \rightarrow c \quad \text { and } \quad\left\|I^{\prime}\left(u_{n}\right)\right\|_{W_{0}^{1, p}(\Omega)^{*}} \rightarrow 0 \quad \text { as } n \rightarrow \infty
$$

for some $c \in \mathbb{R}$. In view of Proposition 2 and the compactness of the embedding $W_{0}^{1, p}(\Omega) \hookrightarrow L^{p}(\Omega)$, it is sufficient to prove the boundedness of $\left\{u_{n}\right\}$ in $W_{0}^{1, p}(\Omega)$. Then, in view of the inequality

$$
\begin{aligned}
\frac{C_{0}}{p(p-1)}\left\|\nabla u_{n}\right\|_{p}^{p} & \leq \int_{\Omega} G\left(x, \nabla u_{n}\right) d x=I\left(u_{n}\right)+\int_{\Omega} F_{+}\left(x, u_{n}\right) d x \\
& \leq I\left(u_{n}\right)+C\left\|u_{n}\right\|_{p}^{p},
\end{aligned}
$$

it is sufficient to prove the boundedness of $\left\{u_{n}\right\}$ in $L^{p}(\Omega)$. By way of contradiction we may assume that $\left\|u_{n}\right\|_{p} \rightarrow \infty$ as $n \rightarrow \infty$ by choosing a subsequence if necessary. Set $v_{n}:=u_{n} /\left\|u_{n}\right\|_{p}$. The inequality (20) ensures that $\left\{v_{n}\right\}$ is bounded in $W_{0}^{1, p}(\Omega)$. Hence, by choosing a subsequence, we may suppose that $v_{n} \rightarrow v_{0}$ in $W_{0}^{1, p}(\Omega)$ and $v_{n} \rightarrow v_{0}$ in $L^{p}(\Omega)$ for some $v_{0}$.

First, we see that $v_{0} \geq 0$ for a.e. $x \in \Omega$. Indeed, by taking $-\left(u_{n}\right)_{-}$as a test function, we have

$$
\begin{aligned}
o(1)\left\|\nabla\left(u_{n}\right)_{-}\right\|_{p} & =\left\langle I^{\prime}\left(u_{n}\right)_{,}-\left(u_{n}\right)_{-}\right\rangle \\
& =\int_{\Omega} A\left(x, \nabla u_{n}\right)\left(-\nabla\left(u_{n}\right)_{-}\right) d x \geq \frac{C_{0}}{p-1}\left\|\nabla\left(u_{n}\right)_{-}\right\|_{p}^{p} .
\end{aligned}
$$

Because $p>1$, we have $\left\|\nabla\left(u_{n}\right)_{-}\right\|_{p} \rightarrow 0$ as $n \rightarrow \infty$. Thus $\left(v_{n}\right)_{-} \rightarrow 0$ in $W_{0}^{1, p}(\Omega)$, and hence $\left(v_{0}\right)_{-}=0$ for a.e. $x \in \Omega$.

Now we prove that

$$
\lim _{n \rightarrow \infty} \frac{\left\|f_{+}\left(\cdot, u_{n}\right)-\alpha\left(u_{n}\right)_{-}^{p-1}\right\|_{p^{\prime}}}{\left\|u_{n}\right\|_{p}^{p-1}}=0,
$$

where $p^{\prime}=p /(p-1)$. Fix an arbitrary $\varepsilon>0$. It follows from condition (ii) of Assumption $(f)$ that there exists a $C_{\varepsilon}>0$ such that

$$
\left|f(x, u)-\alpha u^{p-1}\right| \leq \varepsilon|u|^{p-1}+C_{\varepsilon} \quad \text { for every } u \geq 0 \text {, a.e. } x \in \Omega .
$$

Then we obtain

$$
\int_{\Omega}\left|f_{+}\left(x, u_{n}\right)-\alpha\left(u_{n}\right)_{+}^{p-1}\right|^{p^{\prime}} d x \leq 2^{p^{\prime}-1}\left(\varepsilon^{p^{\prime}-1}\left\|\left(u_{n}\right)_{+}\right\|_{p}^{p}+C_{\varepsilon}^{p^{\prime}-1}|\Omega|\right) .
$$


Since we are assuming that $\left\|u_{n}\right\|_{p} \rightarrow \infty$ as $n \rightarrow \infty$, this shows that

$$
\lim _{n \rightarrow \infty}\left\|f_{+}\left(\cdot, u_{n}\right)-\alpha\left(u_{n}\right)_{+}^{p-1}\right\|_{p^{\prime}} /\left\|u_{n}\right\|_{p}^{p-1}=0,
$$

because $\varepsilon>0$ is arbitrary.

Here we recall the following result proved in Lemma 19:

(22) $\lim _{n \rightarrow \infty} \int_{\Omega} \frac{\tilde{a}\left(x,\left|\nabla u_{n}\right|\right) \nabla u_{n}}{\left\|u_{n}\right\|_{p}^{p-1}} \nabla\left(v_{n}-v_{0}\right) d x=\lim _{n \rightarrow \infty} \int_{\Omega} \frac{\tilde{a}\left(x,\left|\nabla u_{n}\right|\right) \nabla u_{n}}{\left\|u_{n}\right\|_{p}^{p-1}} \nabla \varphi d x=0$

for every $\varphi \in W_{0}^{1, p}(\Omega)$. Thus, by considering

$$
o(1)=\frac{\left\langle I^{\prime}\left(u_{n}\right), v_{n}-v_{0}\right\rangle}{\left\|u_{n}\right\|_{p}^{p-1}}=\int_{\Omega} a_{\infty}(x)\left|\nabla v_{n}\right|^{p-2} \nabla v_{n} \nabla\left(v_{n}-v_{0}\right) d x+o(1),
$$

and using Proposition 2, we see that $v_{n}$ converges strongly to $v_{0}$ in $W_{0}^{1, p}(\Omega)$. Hence, by passing to the limit in $o(1)=\left\langle I^{\prime}\left(u_{n}\right), \varphi\right\rangle /\left\|u_{n}\right\|_{p}^{p-1}$ for any $\varphi \in W_{0}^{1, p}(\Omega)$ and by noting (21) and (22), we infer that $v_{0}$ is a nontrivial solution of

$$
-\operatorname{div}\left(a_{\infty}|\nabla u|^{p-2} \nabla u\right)=\alpha|u|^{p-2} u \quad \text { in } \Omega, \quad u=0 \quad \text { on } \partial \Omega
$$

(note that $\left\|v_{0}\right\|_{p}=1$ and $v_{0} \geq 0$ for a.e. $x \in \Omega$ ). Since $v_{0} \geq 0$ for a.e. $x \in \Omega, v$ is a positive solution of (17) with $\lambda=\alpha$ (see Remark 3). This implies that $\alpha=\lambda_{1}\left(a_{\infty}\right)$, because (17) has no positive solutions if $\lambda \neq \lambda_{1}\left(a_{\infty}\right)$. It contradicts the hypothesis $\alpha \neq \lambda_{1}\left(a_{\infty}\right)$. Hence $\left\|u_{n}\right\|_{p}$ is bounded, which completes the proof.

Lemma 29. Assume ( $\mathrm{AH})$ and $\alpha<\lambda_{1}\left(a_{\infty}\right)$. Then $I$ is coercive, bounded from below and weakly lower semicontinuous (wlsc) on $W_{0}^{1, p}(\Omega)$.

Proof. Because $\alpha<\lambda_{1}\left(a_{\infty}\right)$, we can take sufficiently small constants $\varepsilon>0$ and $0<\delta<1$ satisfying

$$
(1-\delta)\left(\lambda_{1}\left(a_{\infty}\right)-\varepsilon\right)>\alpha+\varepsilon
$$

By condition (ii) of Assumption $(f)$, there exists a $C>0$ such that

$$
\left|F_{+}(x, u)\right| \leq(\alpha+\varepsilon) \frac{u^{p}}{p}+C
$$

for every $u \geq 0$ and a.e. $x \in \Omega$. Due to Proposition 24 and the definition of $\mu_{1}(A, r)$, there exists an $R>0$ such that, for every $u \in W_{0}^{1, p}(\Omega)$ with $\|u\|_{p} \geq R$,

$$
\int_{\Omega} G(x, \nabla u) d x \geq \mu_{1}\left(A,\|u\|_{p}\right) \geq \frac{\lambda_{1}\left(a_{\infty}\right)-\varepsilon}{p}\|u\|_{p}^{p} .
$$

Hence, for every $u \in W_{0}^{1, p}(\Omega)$ with $\|u\|_{p} \geq R$, we obtain 


$$
\begin{aligned}
I(u) & \geq \frac{(1-\delta)\left(\lambda_{1}\left(a_{\infty}\right)-\varepsilon\right)}{p}\|u\|_{p}^{p}+\frac{\delta C_{0}}{p(p-1)}\|\nabla u\|_{p}^{p}-\frac{\alpha+\varepsilon}{p}\left\|u_{+}\right\|_{p}^{p}-C|\Omega| \\
& \geq \frac{\delta C_{0}}{p(p-1)}\|\nabla u\|_{p}^{p}-C|\Omega|
\end{aligned}
$$

by (2), (23), and (24), where $u_{+}:=\max \{0, u\}$. This yields that $I$ is coercive. Moreover, because $I$ is bounded from below on $B_{p}(R)$, we see that $I$ is bounded from below on $W_{0}^{1, p}(\Omega)$. Since $J$ is wlsc (see the proof of Proposition 5) and $W_{0}^{1, p}(\Omega) \hookrightarrow L^{p}(\Omega)$ is compact, $I$ is wlsc on $W_{0}^{1, p}(\Omega)$.

Lemma 30. Assume (AH0) and $\alpha_{0}<\lambda_{1}\left(a_{0}\right)$. Let $p<q \leq p^{*}$, where $p^{*}=$ $N p /(N-p)$ if $N>p$ and $p^{*}=+\infty$ if $N \leq p$. Then there exists $\rho_{0}>0$ such that

$$
\inf \left\{I(u):\|u\|_{q}=\rho\right\}>0 \text { for every } 0<\rho<\rho_{0} .
$$

Proof. Because $\alpha_{0}<\lambda_{1}\left(a_{0}\right)$, we can take some sufficiently small $\varepsilon>0$ and $0<\delta<1$ satisfying

$$
(1-\delta)\left(\lambda_{1}\left(a_{0}\right)-\varepsilon\right)>\alpha_{0}+\varepsilon .
$$

According to Proposition 17, there exists an $r_{0}>0$ such that

$$
\frac{\mu_{1}(A, r)}{r^{p}} \geq \frac{\lambda_{1}\left(a_{0}\right)-\varepsilon}{p} \text { for every } 0<r<r_{0} .
$$

In addition, Assumption $(f)$ guarantees the existence of $D_{q}>0$ satisfying

$$
F_{+}(x, u) \leq \frac{\alpha_{0}+\varepsilon}{p} u^{p}+D_{q} u^{q} \quad \text { for every } u \geq 0, \text { a.e. } x \in \Omega .
$$

Because $W_{0}^{1, p}(\Omega) \hookrightarrow L^{q}(\Omega)$ is continuous, we can take a positive constant $C_{q}$ such that $\|u\|_{q} \leq C_{q}\|\nabla u\|_{p}$ for every $W_{0}^{1, p}(\Omega)$. We choose a positive constant $\rho$ satisfying

$$
\rho<\min \left\{r_{0}|\Omega|^{1 / q-1 / p},\left(\frac{\delta C_{0}}{2 p(p-1) D_{q} C_{q}^{p}}\right)^{1 /(q-p)}\right\}=: \rho_{0} .
$$

Note that $\|u\|_{p}<r_{0}$ if $\|u\|_{q}=\rho$, by Hölder's inequality and (28). Therefore, for every $\|u\|_{q}=\rho$, we have

$$
\begin{aligned}
I(u) & =(1-\delta) \int_{\Omega} G(x, \nabla u) d x+\delta \int_{\Omega} G(x, \nabla u) d x-\int_{\Omega} F_{+}(x, u) d x \\
& \geq(1-\delta) \frac{\mu_{1}\left(A,\|u\|_{p}\right)}{\|u\|_{p}^{p}}\|u\|_{p}^{p}+\frac{\delta C_{0}}{p(p-1)}\|\nabla u\|_{p}^{p}-\frac{\alpha_{0}+\varepsilon}{p}\left\|u_{+}\right\|_{p}^{p}-D_{q}\left\|u_{+}\right\|_{q}^{q} \\
& \geq \frac{1}{p}\left\{(1-\delta)\left(\lambda_{1}\left(a_{0}\right)-\varepsilon\right)-\alpha_{0}-\varepsilon\right\}\|u\|_{p}^{p}+\left(\frac{\delta C_{0}}{p(p-1) C_{q}^{p}}-D_{q}\|u\|_{q}^{q-p}\right)\|u\|_{q}^{p}
\end{aligned}
$$




$$
\geq \frac{\delta C_{0}}{2 p(p-1) C_{q}^{p}} \rho^{p},
$$

by the definition of $\mu_{1}(A, r),(2),(27),(26),(25)$, and (28). This ensures our conclusion.

Proof of Theorem 25. (i) Lemma 29 guarantees the existence of a global minimizer of $I$. Thus it suffices to prove that $\min _{W_{0}^{1, p}(\Omega)} I<0$ to show the existence of a nontrivial critical point of $I$. Choose a positive constant $\varepsilon>0$ such that $\alpha_{0}>$ $\lambda_{1}\left(a_{0}\right)+2 \varepsilon$. Let $\varphi_{a_{0}} \in C^{1}(\bar{\Omega})$ be a positive eigenfunction corresponding to $\lambda_{1}\left(a_{0}\right)$ with $\left\|\varphi_{a_{0}}\right\|_{p}=1$ (refer to the text below (13) and note that (13) is a homogeneous equation). It is easily seen that $\int_{\Omega} \widetilde{G}_{0}\left(x, r \nabla \varphi_{a_{0}}\right) d x / r^{p} \rightarrow 0$ as $r \rightarrow+0$ (refer to the proof of Proposition 17 with $\left\|r \varphi_{a_{0}}\right\|_{p}=r$ ). Hence there exists $r_{0}>0$ such that

$$
\begin{aligned}
\int_{\Omega} G\left(x, r \nabla \varphi_{a_{0}}\right) d x & =\frac{r^{p}}{p} \int_{\Omega} a_{0}(x)\left|\nabla \varphi_{a_{0}}\right|^{p} d x+r^{p} \int_{\Omega} \frac{\widetilde{G}_{0}\left(x, r \nabla \varphi_{a_{0}}\right)}{r^{p}} d x \\
& \leq \frac{\lambda_{1}\left(a_{0}\right)+\varepsilon}{p} r^{p}=\frac{\lambda_{1}\left(a_{0}\right)+\varepsilon}{p}\left\|r \varphi_{a_{0}}\right\|_{p}^{p}
\end{aligned}
$$

for every $0<r<r_{0}$. On the other hand, it follows from part (i) of Assumption $(f)$ that there exists a $\delta>0$ such that

$$
F_{+}(x, u) \geq \frac{\alpha_{0}-\varepsilon}{p} u^{p} \quad \text { for every } u \in[0, \delta], \text { a.e. } x \in \Omega .
$$

Therefore, for every $0<r<\min \left\{r_{0}, \delta /\left\|\varphi_{a_{0}}\right\|_{\infty}\right\}$, we have

$$
I\left(r u_{0}\right) \leq \frac{r^{p}}{p}\left(\lambda_{1}\left(a_{0}\right)+2 \varepsilon-\alpha_{0}\right)\left\|\varphi_{a_{0}}\right\|_{p}^{p}<0,
$$

by (29) and (30) (note $\lambda_{1}\left(a_{0}\right)+2 \varepsilon-\alpha_{0}<0$ ), whence $\min _{W_{0}^{1, p}(\Omega)} I<0$.

(ii) Let $p<q \leq p^{*}$. Then, by Lemma 30, we obtain $\rho>0$ satisfying

$$
\delta_{0}:=\inf \left\{I(u):\|u\|_{q}=\rho\right\}>0 .
$$

Now we claim the existence of $w \in W_{0}^{1, p}(\Omega)$ such that

$$
\|w\|_{q}>\rho \quad \text { and } \quad I(w)<\delta_{0} .
$$

Admitting this claim, we define

$$
c:=\inf _{\gamma \in \Gamma} \max _{t \in[0,1]} I(\gamma(t)), \quad \Gamma:=\left\{\gamma \in C\left([0,1], W_{0}^{1, p}(\Omega)\right): \gamma(0)=0, \gamma(1)=w\right\}
$$

It is obvious that $\Gamma \neq \varnothing$ and $\gamma([0,1]) \cap\left\{u \in W_{0}^{1, p}(\Omega):\|u\|_{q}=\rho\right\} \neq \varnothing$ for every $\gamma \in \Gamma$, since $W_{0}^{1, p}(\Omega) \hookrightarrow L^{q}(\Omega)$ is continuous. Thus the mountain pass theorem guarantees that $c\left(\geq \delta_{0}\right)$ is a nontrivial critical value of $I$ because $I$ satisfies the Palais-Smale condition by Lemma 28. 
Finally, we prove the existence of $w$ satisfying (31). Because $\alpha>\lambda_{1}\left(a_{\infty}\right)$, we can choose a positive constant $\varepsilon_{0}>0$ such that

$$
\alpha>\lambda_{1}\left(a_{\infty}\right)+2 \varepsilon_{0} .
$$

Using item (ii) on page 165 , we can take $\varphi_{a_{\infty}} \in C^{1}(\bar{\Omega})$ be a positive eigenfunction corresponding to $\lambda_{1}\left(a_{\infty}\right)$ with $\left\|\varphi_{a_{\infty}}\right\|_{p}=1$. It follows from Lemma 19(iii) that

$$
\int_{\Omega} \widetilde{G}\left(x, r \nabla \varphi_{a_{\infty}}\right) d x / r^{p} \rightarrow 0
$$

as $r \rightarrow+\infty$ (note that $\left\|r \varphi_{a_{\infty}}\right\|_{p}=r$ ). Hence there exists $R_{0}>0$ such that

$$
\begin{aligned}
\int_{\Omega} G\left(x, r \nabla \varphi_{a_{\infty}}\right) d x & =\frac{r^{p}}{p} \int_{\Omega} a_{\infty}(x)\left|\nabla \varphi_{a_{\infty}}\right|^{p} d x+r^{p} \int_{\Omega} \frac{\widetilde{G}_{0}\left(x, r \nabla \varphi_{a_{\infty}}\right)}{r^{p}} d x \\
& \leq \frac{\lambda_{1}\left(a_{\infty}\right)+\varepsilon_{0}}{p} r^{p}=\frac{\lambda_{1}\left(a_{\infty}\right)+\varepsilon_{0}}{p}\left\|r \varphi_{a_{\infty}}\right\|_{p}^{p}
\end{aligned}
$$

for every $r \geq R_{0}$. In addition, it follows from condition (ii) of Assumption $(f)$ that there exists $D>0$ such that

$$
F_{+}(x, u) \geq \frac{\alpha-\varepsilon_{0}}{p} u^{p}-D \quad \text { for every } u \geq 0, \text { a.e. } x \in \Omega .
$$

Consequently, by (32), (33), and (34), we obtain

$$
I\left(r \varphi_{a_{0}}\right) \leq \frac{r^{p}}{p}\left(\lambda_{1}\left(a_{\infty}\right)+2 \varepsilon_{0}-\alpha\right)\left\|\varphi_{a_{0}}\right\|_{p}^{p}+D|\Omega| \rightarrow-\infty
$$

as $t \rightarrow+\infty$. This implies the existence of $w$ satisfying (31).

4.1. Resonant cases. To consider the resonant cases, we introduce the following hypotheses for

$$
\widetilde{G}(x, y):=\int_{0}^{|y|} \tilde{a}(x, t) t d t \quad \text { and } \quad \widetilde{G}_{0}(x, y):=\int_{0}^{|y|} \tilde{a}_{0}(x, t) t d t,
$$

where $\tilde{a}$ and $\tilde{a}_{0}$ are as in (AH) and (AH0).

(H+) There exist $1 \leq q<p$ and $H_{0}>0$ such that

$$
\begin{array}{rlrl}
\lim _{|y| \rightarrow \infty} \frac{p \widetilde{G}(x, y)-\tilde{a}(x,|y|)|y|^{2}}{|y|^{q}} & =+\infty & \text { for a.e. } x \in \Omega, \\
p \widetilde{G}(x, y)-\tilde{a}(x,|y|)|y|^{2} \geq-H_{0}\left(1+|y|^{q}\right) & \text { for a.e. } x \in \Omega, \text { every } y \in \mathbb{R}^{N}, \\
f(x, t) t-p F(x, t) \geq-H_{0}\left(1+t^{q}\right) & \text { for a.e. } x \in \Omega, \text { every } t \geq 0 .
\end{array}
$$


(H-) There exist $1 \leq q<p$ and $H_{0}>0$ such that

$$
\begin{array}{ll}
\lim _{|y| \rightarrow \infty} \frac{p \widetilde{G}(x, y)-\tilde{a}(x,|y|)|y|^{2}}{|y|^{q}}=-\infty & \text { for a.e. } x \in \Omega, \\
p \widetilde{G}(x, y)-\tilde{a}(x,|y|)|y|^{2} \leq H_{0}\left(1+|y|^{q}\right) & \text { for a.e. } x \in \Omega, \text { every } y \in \mathbb{R}^{N}, \\
f(x, t) t-p F(x, t) \leq H_{0}\left(t^{q}+1\right) & \text { for a.e. } x \in \Omega, \text { every } t \geq 0 .
\end{array}
$$

$(\mathrm{HF}+)$ There exist $1 \leq q<p$ and $H_{0}>0$ such that

$$
\begin{aligned}
p \widetilde{G}(x, y)-\tilde{a}(x,|y|)|y|^{2} & \geq-H_{0}\left(1+|y|^{q}\right) & & \text { for a.e. } x \in \Omega, \text { every } y \in \mathbb{R}^{N}, \\
f(x, t) t-p F(x, t) & \geq-H_{0}\left(1+t^{q}\right) & & \text { for every } t \geq 0, \text { a.e. } x \in \Omega, \\
\lim _{t \rightarrow+\infty} \frac{f(x, t) t-p F(x, t)}{t^{q}} & =+\infty & & \text { for a.e. } x \in \Omega .
\end{aligned}
$$

(HF-) There exist $1 \leq q<p$ and $H_{0}>0$ such that

$$
\begin{aligned}
p \widetilde{G}(x, y)-\tilde{a}(x,|y|)|y|^{2} & \leq H_{0}\left(1+|y|^{q}\right) & & \text { for a.e. } x \in \Omega, \text { every } y \in \mathbb{R}^{N}, \\
f(x, t) t-p F(x, t) & \leq H_{0}\left(1+t^{q}\right) & & \text { for every } t \geq 0, \text { a.e. } x \in \Omega, \\
\lim _{t \rightarrow+\infty} \frac{f(x, t) t-p F(x, t)}{t^{q}} & =-\infty & & \text { for a.e. } x \in \Omega .
\end{aligned}
$$

(H0+) There exist $p \leq r<p^{*}$ and $H_{0}>0$ such that

$$
\begin{aligned}
\lim _{|y| \rightarrow 0} \frac{p \widetilde{G}_{0}(x, y)-\tilde{a}_{0}(x,|y|)|y|^{2}}{|y|^{r}} & =+\infty & & \text { for a.e. } x \in \Omega, \\
p \widetilde{G}(x, y)-\tilde{a}(x,|y|)|y|^{2} & \geq-H_{0}|y|^{r} & & \text { for a.e. } x \in \Omega, \text { every }|y| \leq 1, \\
f(x, t) t-p F(x, t) & \geq-H_{0} t^{r} & & \text { for a.e. } x \in \Omega, \text { every } t \in[0,1] .
\end{aligned}
$$

(H0-) There exist $p \leq r<p^{*}$ and $H_{0}>0$ such that

$$
\begin{aligned}
\lim _{|y| \rightarrow 0} \frac{p \widetilde{G}_{0}(x, y)-\tilde{a}_{0}(x,|y|)|y|^{2}}{|y|^{r}} & =-\infty & & \text { for a.e. } x \in \Omega, \\
p \widetilde{G}(x, y)-\tilde{a}(x,|y|)|y|^{2} & \leq H_{0}|y|^{r} & & \text { for a.e. } x \in \Omega, \text { every }|y| \leq 1, \\
f(x, t) t-p F(x, t) & \leq H_{0} t^{r} & & \text { for a.e. } x \in \Omega, \text { every } t \in[0,1] .
\end{aligned}
$$

(HF0+) There exist $p \leq r<p^{*}$ and $H_{0}>0$ such that

$$
\begin{aligned}
p \widetilde{G}_{0}(x, y)-\tilde{a}_{0}(x,|y|)|y|^{2} & \geq-H_{0}|y|^{r} & & \text { for a.e. } x \in \Omega, \text { every }|y| \leq 1, \\
f(x, t) t-p F(x, t) & \geq-H_{0} t^{r} & & \text { for every } t \in[0,1], \text { a.e. } x \in \Omega, \\
\lim _{t \rightarrow+0} \frac{f(x, t) t-p F(x, t)}{t^{r}} & =+\infty & & \text { for a.e. } x \in \Omega .
\end{aligned}
$$


(HF0-) There exist $p \leq r<p^{*}$ and $H_{0}>0$ such that

$$
\begin{array}{rlrl}
p \widetilde{G}_{0}(x, y)-\tilde{a}_{0}(x,|y|)|y|^{2} & \leq H_{0}|y|^{r} & & \text { for a.e. } x \in \Omega, \text { every }|y| \leq 1, \\
f(x, t) t-p F(x, t) & \leq H_{0} t^{r} & \text { for every } t \in[0,1], \text { a.e. } x \in \Omega, \\
\lim _{t \rightarrow+0} \frac{f(x, t) t-p F(x, t)}{t^{r}} & =-\infty & \text { for a.e. } x \in \Omega .
\end{array}
$$

Theorem 31. Let Assumption $(f)$, (AH0), and (AH) hold. If any of the following conditions is satisfied, (P) has at least one positive solution.

(i) $\alpha_{0}>\lambda_{1}\left(a_{0}\right), \alpha=\lambda_{1}\left(a_{\infty}\right)$, and $(\mathrm{HF}+)$ or $(\mathrm{H}+)$.

(ii) $\alpha_{0}<\lambda_{1}\left(a_{0}\right), \alpha=\lambda_{1}\left(a_{\infty}\right)$, and (HF-) or $(\mathrm{H}-)$.

(iii) $\alpha_{0}=\lambda_{1}\left(a_{0}\right), \alpha<\lambda_{1}\left(a_{\infty}\right)$, and (HF0+) or (H0+).

(iv) $\alpha_{0}=\lambda_{1}\left(a_{0}\right), \alpha>\lambda_{1}\left(a_{\infty}\right)$, and (HF0-) or (H0-).

(v) $\alpha_{0}=\lambda_{1}\left(a_{0}\right), \alpha=\lambda_{1}\left(a_{\infty}\right),(\mathrm{HF} 0+)$ or $(\mathrm{H} 0+)$, and $(\mathrm{HF}+)$ or $(\mathrm{H}+)$.

(vi) $\alpha_{0}=\lambda_{1}\left(a_{0}\right), \alpha=\lambda_{1}\left(a_{\infty}\right)$, (HF0-) or (H0-), and (HF-) or (H-).

The rest of this section is devoted to the proof of this theorem, which involves some preparatory steps.

The singly resonant case. Set $f_{ \pm n}(x, t):=f(x, t) \pm \frac{p}{n}|t|^{p-2} t$ and define approximate functionals on $W_{0}^{1, p}(\Omega)$ by

$$
I_{ \pm n}(u):=\int_{\Omega} G(x, \nabla u) d x-\int_{\Omega}\left(F_{ \pm n}\right)_{+}(x, u) d x=I(u) \mp \frac{1}{n}\left\|u_{+}\right\|_{p}^{p} .
$$

From now on, assume $f$ satisfies Assumption $(f)$. Take first the case $\alpha=\lambda_{1}\left(a_{\infty}\right)$. Lemma 32. If either $(\mathrm{H}+)$ or $(\mathrm{HF}+)$ (resp. either $(\mathrm{H}-)$ or $(\mathrm{HF}-))$ hold and $\left\{u_{n}\right\}$ satisfies

$$
\begin{array}{rll}
\sup _{n \in \mathbb{N}} I_{ \pm n}\left(u_{n}\right)<+\infty & \text { and } & \lim _{n \rightarrow \infty}\left\|I_{ \pm n}^{\prime}\left(u_{n}\right)\right\|_{W_{0}^{1, p}(\Omega)^{*}}=0 \\
\text { resp. } & \inf _{n \in \mathbb{N}} I_{ \pm n}\left(u_{n}\right)>-\infty \quad \text { and } & \left.\lim _{n \rightarrow \infty}\left\|I_{ \pm n}^{\prime}\left(u_{n}\right)\right\|_{W_{0}^{1, p}(\Omega)^{*}}=0\right),
\end{array}
$$

then $\left\{u_{n}\right\}$ is bounded in $W_{0}^{1, p}(\Omega)$.

Proof. The boundedness of $\left\|u_{n}\right\|_{p}$ guarantees that $\left\|u_{n}\right\|$ is bounded, since

$$
o(1)\left\|u_{n}\right\|=\left\langle I_{ \pm n}^{\prime}\left(u_{n}\right), u_{n}\right\rangle \geq \frac{C_{0}}{p-1}\left\|u_{n}\right\|^{p}-C\left(1+\left\|u_{n}\right\|_{p}^{p}\right) \mp \frac{1}{n}\left\|\left(u_{n}\right)_{+}\right\|_{p}^{p}
$$

for some $C>0$ independent of $n$. So, by way of contradiction, we assume that $\left\|u_{n}\right\|_{p} \rightarrow \infty$ as $n \rightarrow \infty$. Then, by the same argument as in Lemma 28, we see that $v_{n}:=u_{n} /\left\|u_{n}\right\|_{p}$ has a subsequence strongly converging to a positive solution $v_{0}$ of

$$
-\operatorname{div}\left(a_{\infty}|\nabla u|^{p-2} \nabla u\right)=\alpha|u|^{p-2} u \quad \text { in } \Omega, \quad u=0 \quad \text { on } \partial \Omega .
$$


If $\alpha \neq \lambda_{1}\left(a_{\infty}\right)$, we have a contradiction, because (35) does not have a positive solution except when $\lambda=\lambda_{1}\left(a_{\infty}\right)$. So we may assume that $\alpha=\lambda_{1}\left(a_{\infty}\right)$ and $v_{0}=\varphi_{a_{\infty}}$ (note $\left\|v_{0}\right\|_{p}=1$ ). For simplicity, we still denote the subsequence under discussion by $\left\{v_{n}\right\}$. Thus $u_{n}(x) \rightarrow \infty$ as $n \rightarrow \infty$ for a.e. $x \in \Omega$ (note $v_{0}=\varphi_{a_{\infty}}>0$ in $\Omega$ ).

Assume $(\mathrm{HF}+)$ or $(\mathrm{HF}-)$. We show that

$$
I:=\int_{\Omega} \frac{f_{+}\left(x, u_{n}\right) u_{n}-p F_{+}\left(x, u_{n}\right)}{\left\|u_{n}\right\|_{p}^{q}} d x \rightarrow \pm \infty,
$$

where the sign on $\infty$ matches ( $\mathrm{HF} \pm$ ) and $q$ is a constant as in $(\mathrm{HF} \pm)$. Indeed, it follows from (HF+) that $\left(f_{+}(x, t) t-p F_{+}(x, t)\right) / t^{q}$ is bounded from below on $\Omega \times[1,+\infty)$. Therefore, since $u_{n}(x) \rightarrow \infty$ for a.e. $x \in \Omega$, we have (36) if (HF+) holds, by applying Fatou's lemma to the inequality

$$
I \geq \int_{u_{n}(x) \geq 1} \frac{f_{+}\left(x, u_{n}\right) u_{n}-p F_{+}\left(x, u_{n}\right)}{u_{n}^{q}} v_{n}^{q} d x-\frac{2 H_{0}}{\left\|u_{n}\right\|_{p}^{p}}|\Omega|,
$$

where $H_{0}>0$ is a constant as in (HF+). The case of (HF-) is handled by the same argument, with $-f$ instead of $f$. This shows (36).

Furthermore, by Hölder's inequality, we have

$$
\begin{aligned}
I I & :=\int_{\Omega} \frac{p \widetilde{G}\left(x, \nabla u_{n}\right)-\tilde{a}\left(x,\left|\nabla u_{n}\right|\right)\left|\nabla u_{n}\right|^{2}}{\left\|u_{n}\right\|_{p}^{q}} d x \\
& \leq H_{0} \int_{\Omega}\left(\left|\nabla v_{n}\right|^{q}+\frac{1}{\left\|u_{n}\right\|_{p}^{q}}\right) d x \leq H_{0}\left\|\nabla v_{n}\right\|_{p}^{q}|\Omega|^{(p-q) / p}+o(1) \\
& \leq H_{0}\left\|\nabla v_{0}\right\|_{p}^{q}|\Omega|^{(p-q) / p}+o(1)
\end{aligned}
$$

in the case of (HF-), because $v_{n} \rightarrow v_{0}$ in $W_{0}^{1, p}(\Omega)$, where $q \in[1, p)$ and $H_{0}>0$ are constants as in (HF-). Similarly, we obtain

$$
I I \geq-H_{0}\left\|\nabla v_{0}\right\|_{p}^{q}|\Omega|^{(p-q) / p}+o(1)
$$

in the case of $(\mathrm{HF}+)$.

Hence we have a contradiction because of (36), (37) or (38) by taking the limit inferior or superior in the equality

$$
\frac{p I_{ \pm n}\left(u_{n}\right)-\left\langle I_{ \pm n}^{\prime}\left(u_{n}\right), u_{n}\right\rangle}{\left\|u_{n}\right\|_{p}^{q}}=I I+I .
$$

Assume $(\mathrm{H}+)$ or $(\mathrm{H}-)$. Because $v_{0}$ is a positive solution of (35), we have $\left|\nabla u_{n}(x)\right| \rightarrow \infty$ as $n \rightarrow \infty$ for a.e. $x \in \Omega_{0}:=\left\{x^{\prime} \in \Omega:\left|\nabla v_{0}\left(x^{\prime}\right)\right| \neq 0\right\}$. Because $\left|\Omega_{0}\right|>0$, we can show, by an argument similar to the one used for $f$, that

$$
\int_{\Omega} \frac{p \widetilde{G}\left(x, \nabla u_{n}\right)-\tilde{a}\left(x,\left|\nabla u_{n}\right|\right)\left|\nabla u_{n}\right|^{2}}{\left\|u_{n}\right\|_{p}^{q}} d x \rightarrow \pm \infty,
$$


where again the sign matches that of $(\mathrm{H} \pm)$. In addition, we easily obtain that

$$
\pm \int_{\Omega} \frac{f_{+}\left(x, u_{n}\right) u_{n}-p F_{+}\left(x, u_{n}\right)}{\left\|u_{n}\right\|_{p}^{q}} d x \geq-H_{0}\left\|v_{n}\right\|_{q}^{q}+o(1)=-H_{0}\left\|v_{0}\right\|_{q}^{q}+o(1)
$$

(again, the sign matches). Hence we have a contradiction by considering the limit of $\left(p I_{ \pm n}\left(u_{n}\right)-\left\langle I_{ \pm n}^{\prime}\left(u_{n}\right), u_{n}\right\rangle\right) /\left\|u_{n}\right\|_{p}^{q}$.

Proof of Theorem 31(i). Because $\alpha_{0}>\lambda_{1}\left(a_{0}\right)$, there exists an $n_{0} \in \mathbb{N}$ such that $\alpha_{0}-p / n_{0}>\lambda_{1}\left(a_{0}\right)$. Note that $f_{-n}(x, t) / t^{p-1} \rightarrow \alpha_{0}-p / n>\lambda_{1}\left(a_{0}\right)$ as $t \rightarrow+0$ for $n \geq n_{0}$ and $f_{-n}(x, t) / t^{p-1} \rightarrow \alpha-p / n=\lambda_{1}\left(a_{\infty}\right)-p / n<\lambda_{1}\left(a_{\infty}\right)$ as $t \rightarrow+\infty$. Hence, by using the proof of Theorem 25(i) to $f_{-n}$, we can find a global minimizer $u_{n}$ of $I_{-n}$ with $I_{-n}\left(u_{n}\right)<0$ for each $n \geq n_{0}$. Here we remark that $\sup _{n \geq n_{0}} I_{-n}\left(u_{n}\right)<$ 0 . In fact, for every $n \geq n_{0}$, we have

$$
I_{-n}\left(u_{n}\right) \leq I_{-n}\left(u_{n_{0}}\right)=I\left(u_{n_{0}}\right)+\frac{1}{n}\left\|u_{n_{0}}\right\|_{p}^{p} \leq I\left(u_{n_{0}}\right)+\frac{1}{n_{0}}\left\|u_{n_{0}}\right\|_{p}^{p}=I_{-n_{0}}\left(u_{n_{0}}\right)<0,
$$

where, in the first inequality, we use the fact that $u_{n}$ is a global minimizer of $I_{-n}$. Now, due to Lemma 32, we see that $\left\{u_{n}\right\}$ is bounded in $W_{0}^{1, p}(\Omega)$. Therefore,

$$
\left\|I^{\prime}\left(u_{n}\right)\right\|_{W_{0}^{1, p}(\Omega)^{*}}=\left\|I^{\prime}\left(u_{n}\right)-I_{-n}^{\prime}\left(u_{n}\right)\right\|_{W_{0}^{1, p}(\Omega)^{*}} \leq \frac{p}{n \lambda_{1}\left(-\Delta_{p}\right)^{p}}\left\|u_{n}\right\|^{p-1} \rightarrow 0
$$

as $n \rightarrow \infty$, where $\lambda_{1}\left(-\Delta_{p}\right)$ is the first eigenvalue of $-\Delta_{p}$. Since $I$ is bounded on a bounded set, we may assume that $\left\{u_{n}\right\}$ is a bounded Palais-Smale sequence of $I$. Because $I$ satisfies the bounded Palais-Smale condition (see Proposition 2), $u_{n}$ has a subsequence converging to some $v_{0}$ in $W_{0}^{1, p}(\Omega)$. It is clear that $I\left(v_{0}\right) \leq$ $\sup _{n \geq n_{0}} I_{-n}\left(u_{n}\right)=I_{-n_{0}}\left(u_{n_{0}}\right)<0$, and so $v_{0}$ is a nontrivial critical point of $I$.

Proof of Theorem 31(ii). Using Lemma 30 and $\alpha_{0}<\lambda_{1}\left(a_{0}\right)$, we can choose $q_{0} \in\left(p, p^{*}\right]$ and $\rho>0$ such that $\inf \left\{I(u):\|u\|_{q_{0}}=\rho\right\}>0$. Since $I_{+n}(u) \geq$ $I(u)-\|u\|_{q_{0}}^{p}|\Omega|^{1-p / q_{0}} / n$ for every $u \in W_{0}^{1, p}(\Omega)$, we can take $n_{0} \in \mathbb{N}$ such that $\alpha_{0}+p / n_{0}<\lambda_{1}\left(a_{0}\right)$ and $\delta_{0}:=\inf \left\{I_{+n_{0}}(u):\|u\|_{q_{0}}=\rho\right\}>0$. Hence, for every $n \geq n_{0}$, we have $\inf \left\{I_{+n}(u):\|u\|_{q_{0}}=\rho\right\} \geq \delta_{0}$, because $I_{+n}(u) \geq I_{+n_{0}}(u)$ for every $n \geq n_{0}$ and $u \in W_{0}^{1, p}(\Omega)$. By noting that $f_{+n}(x, t) / t^{p-1} \rightarrow \alpha+p / n>\alpha=\lambda_{1}\left(a_{\infty}\right)$ as $t \rightarrow+\infty$, and applying Lemma 28 to $f_{+n}$ instead of $f, I_{+n}$ satisfies the Palais-Smale condition. Therefore, the proof of Theorem 25(ii) implies that, for every $n \geq n_{0}$, there exists a critical point $u_{n} \in W_{0}^{1, p}(\Omega)$ of $I_{+n}$ such that $I_{+n}\left(u_{n}\right) \geq \delta_{0}$. According to Lemma 32, $\left\{u_{n}\right\}$ is bounded in $W_{0}^{1, p}(\Omega)$. Thus, because we have a bounded Palais-Smale sequence of $I$ due to a similar reason as in the case of (i), we can obtain a nontrivial critical point of $I$ (note that $\inf _{n \geq n_{0}} I\left(u_{n}\right) \geq \inf _{n \geq n_{0}} I_{+n}\left(u_{n}\right) \geq \delta_{0}>0$ ).

We next turn to the case where $\alpha_{0}=\lambda_{1}\left(a_{0}\right)$. 
Lemma 33. Assume (H0-) or ( $\mathrm{HF} 0-)$ (resp. $(\mathrm{H} 0+)$ or $(\mathrm{HF} 0+))$. Let $u_{n} \neq 0$ be an element of $W_{0}^{1, p}(\Omega)$ satisfying $I_{ \pm n}^{\prime}\left(u_{n}\right)=0$ for every $n \in \mathbb{N}$ and $\inf _{n} I_{ \pm n}\left(u_{n}\right) \geq 0$ (resp. $\left.\sup _{n} I_{ \pm n}\left(u_{n}\right) \leq 0\right)$. Then $\liminf _{n \rightarrow \infty}\left\|u_{n}\right\|_{p}>0$.

Proof. By way of contradiction, we assume that $\lim _{n \rightarrow \infty}\left\|u_{n}\right\|_{p}=0$ by choosing a subsequence. Note that the boundedness of $\left\|u_{n}\right\|_{p}$ yields that $\left\|u_{n}\right\|$ and $\left\|u_{n}\right\| /\left\|u_{n}\right\|_{p}$ are bounded in view of

$$
o(1)\left\|u_{n}\right\|=\left\langle I_{ \pm n}^{\prime}\left(u_{n}\right), u_{n}\right\rangle \geq \frac{C_{0}}{p-1}\left\|u_{n}\right\|^{p}-C\left(1+\left\|\left(u_{n}\right)_{+}\right\|_{p}^{p}\right) \mp \frac{p}{n}\left\|\left(u_{n}\right)_{+}\right\|_{p}^{p}
$$

for some $C>0$ independent of $n$. Then, since $u_{n}$ is a positive solution of

$$
-\operatorname{div}(A(x, \nabla u))=f_{ \pm n}\left(x, u_{n}\right) \quad \text { in } \Omega
$$

(refer to Remarks 3 and 27), it follows from Proposition 4 that $u_{n} \rightarrow 0$ in $C^{1}(\bar{\Omega})$ (note that $\left|\left(f_{ \pm n}\right)_{+}(x, t)\right| \leq C t_{+}^{p-1}$ (see Assumption $\left.(f)\right)$ and $u_{n} \rightarrow 0$ in $\left.L^{p}(\Omega)\right)$. Therefore, we may assume that $\left\|u_{n}\right\|_{C^{1}(\bar{\Omega})} \leq 1$ by considering a sufficiently large $n$. Since $\left|f_{ \pm n}\left(x,\left\|u_{n}\right\|_{p} t\right) /\left\|u_{n}\right\|_{p}^{p-1}\right| \leq C t^{p}$ for every $t \geq 0$, a.e. $x \in \Omega(C>0$ independent of $n$; see Assumption $(f)$ and (39)), by a similar argument to Theorem 13, we see that $v_{n}:=u_{n} /\left\|u_{n}\right\|_{p}$ has a subsequence converging to a positive solution $v_{0}$ in $C^{1}(\bar{\Omega})$ of

$$
-\operatorname{div}\left(a_{0}(x)|\nabla u|^{p-2} \nabla u\right)=\alpha_{0}|u|^{p-2} u \quad \text { in } \Omega, \quad u=0 \quad \text { on } \partial \Omega .
$$

If $\alpha_{0} \neq \lambda_{1}\left(a_{0}\right)$, we have a contradiction because (13) does not have a positive solution unless $\lambda=\lambda_{1}\left(a_{0}\right)$. So we may assume that $\alpha_{0}=\lambda_{1}\left(a_{0}\right)$ and $v_{0}=\varphi_{a_{0}}$ (note $\left.\left\|v_{0}\right\|_{p}=1\right)$. For simplicity, we still denote the subsequence under discussion by $\left\{v_{n}\right\}$.

Assume $(\mathrm{H} 0+)$ or $(\mathrm{H} 0-)$. Then we can prove that

$$
I:=\int_{\Omega} \frac{p \widetilde{G}_{0}\left(x, \nabla u_{n}\right)-\tilde{a}_{0}\left(x,\left|\nabla u_{n}\right|\right)\left|\nabla u_{n}\right|^{2}}{\left\|u_{n}\right\|_{p}^{r}} d x \rightarrow \pm \infty
$$

(signs match), where $r \in\left[p, p^{*}\right)$ is a constant as in (H0+) or (H0-). Indeed, because $\left\|\nabla v_{0}\right\|_{p}>0$, we can choose a constant $\varepsilon_{0}>0$ such that $\mid\left\{x \in \Omega:\left|\nabla v_{0}\right|>\right.$ $\left.2 \varepsilon_{0}\right\} \mid>0$. With this $\varepsilon_{0}$, we have under assumption $(\mathrm{H} 0+)$

$$
\begin{aligned}
I & \geq \int_{\left|\nabla v_{n}\right|>\varepsilon_{0}} \frac{p \widetilde{G}_{0}\left(x, \nabla u_{n}\right)-\tilde{a}_{0}\left(x,\left|\nabla u_{n}\right|\right)\left|\nabla u_{n}\right|^{2}}{\left|\nabla u_{n}\right|^{r}}\left|\nabla v_{n}\right|^{r} d x-\int_{\left|\nabla v_{n}\right| \leq \varepsilon_{0}} H_{0}\left|\nabla v_{n}\right|^{r} d x \\
& \geq \int_{\left|\nabla v_{n}\right|>\varepsilon_{0}} \frac{p \widetilde{G}_{0}\left(x, \nabla u_{n}\right)-\tilde{a}_{0}\left(x,\left|\nabla u_{n}\right|\right)\left|\nabla u_{n}\right|^{2}}{\left|\nabla u_{n}\right|^{r}}\left|\nabla v_{n}\right|^{r} d x-\varepsilon_{0}^{r} H_{0}|\Omega|,
\end{aligned}
$$

where $H_{0}$ is a positive constant as in $(\mathrm{H} 0+)$. Hence, applying Fatou's lemma, our claim is shown, because the Lebesgue measure of $\left\{x \in \Omega:\left|\nabla v_{0}\right|>2 \varepsilon_{0}\right\}$ is positive. Similarly, by considering $\tilde{a}_{0}\left(x,\left|\nabla u_{n}\right|\right)\left|\nabla u_{n}\right|^{2}-p \widetilde{G}_{0}\left(x, \nabla u_{n}\right)$, we can prove (41) under $(\mathrm{H} 0-)$. 
On the other hand, by using $(\mathrm{H} 0+)$ or $(\mathrm{H} 0-)$, we obtain

$$
\begin{aligned}
\pm I I & := \pm \int_{\Omega} \frac{f_{+}\left(x, u_{n}\right) u_{n}-p F_{+}\left(x, u_{n}\right)}{\left\|u_{n}\right\|_{p}^{r}} d x \geq-H_{0} \int_{\Omega}\left(v_{n}\right)_{+}^{r} d x \\
& \geq-H_{0}\left\|v_{n}\right\|_{r}^{r}=-H_{0}\left\|v_{0}\right\|_{r}^{r}+o(1)
\end{aligned}
$$

(note that $\left\|u_{n}\right\|_{C^{1}(\bar{\Omega})} \leq 1$ and $v_{n} \rightarrow v_{0}$ in $C^{1}(\bar{\Omega})$ ). Now set $\Psi_{n}=I_{ \pm n}$. Since

$$
\pm(I+I I)= \pm \frac{p \Psi_{n}\left(u_{n}\right)-\left\langle\Psi_{n}^{\prime}\left(u_{n}\right), u_{n}\right\rangle}{\left\|u_{n}\right\|_{p}^{r}}= \pm \frac{p \Psi_{n}\left(u_{n}\right)}{\left\|u_{n}\right\|_{p}^{r}} \leq 0
$$

if $\sup _{n}\left( \pm I_{ \pm}\left(u_{n}\right)\right) \leq 0$ (where the signs match throughout), we obtain a contradiction with (41) and (42) by taking the limit superior or inferior in (43).

Assume (HF0+) or (HF0-). As in the argument for $I$ in the case of $(\mathrm{H} 0 \pm)$, we can show that

$$
\int_{\Omega} \frac{f_{+}\left(x, u_{n}\right) u_{n}-p F_{+}\left(x, u_{n}\right)}{\left\|u_{n}\right\|_{p}^{r}} d x=\int_{v_{n}>0} \frac{f_{+}\left(x, u_{n}\right) u_{n}-p F_{+}\left(x, u_{n}\right)}{\left(u_{n}\right)_{+}^{r}}\left(v_{n}\right)_{+}^{r} d x
$$

the sign matching that of $(\mathrm{HF} 0 \pm)$. Moreover, it is easily seen that

$$
\pm \int_{\Omega} \frac{p \widetilde{G}_{0}\left(x, \nabla u_{n}\right)-\tilde{a}_{0}\left(x,\left|\nabla u_{n}\right|\right)\left|\nabla u_{n}\right|^{2}}{\left\|u_{n}\right\|_{p}^{r}} d x \geq \mp H_{0}\left\|\nabla v_{n}\right\|_{r}^{r}=\mp H_{0}\left\|\nabla v_{0}\right\|_{r}^{r}+o(1) \text {. }
$$

(Note that $\left\|u_{n}\right\|_{C^{1}(\bar{\Omega})} \leq 1$ and $v_{n} \rightarrow v_{0}$ in $C^{1}(\bar{\Omega})$.) Our conclusion follows from a similar argument as before.

Proof of Theorem 31(iii). Let $n_{0} \in \mathbb{N}$ such that $\alpha+p / n_{0}<\lambda_{1}\left(a_{\infty}\right)$. The proof of Theorem 25(i) guarantees that, for every $n \geq n_{0}, I_{+n}$ has a global minimizer $u_{n}$ such that $I_{+n}\left(u_{n}\right)<0$, because $f_{+n}(x, t) / t^{p-1} \rightarrow \alpha_{0}+p / n>\alpha_{0}=\lambda_{1}\left(a_{0}\right)$ as $t \rightarrow+0$ and $f_{+n}(x, t) / t^{p-1} \rightarrow \alpha+p / n<\lambda_{1}\left(a_{\infty}\right)$ as $t \rightarrow+\infty$ if $n \geq n_{0}$. Noting that $I_{+n}(u) \geq I_{+n_{0}}(u)$ for every $u \in W_{0}^{1, p}(\Omega)$ and $n \geq n_{0},\left\{u_{n}\right\}$ is bounded in $W_{0}^{1, p}(\Omega)$ since $I_{+n_{0}}$ is coercive on $W_{0}^{1, p}(\Omega)$ by Lemma 29. Thus $\left\{u_{n}\right\}$ is a bounded Palais-Smale sequence of $I$ by the same argument as in (i). Therefore, $\left\{u_{n}\right\}$ has a convergent subsequence to some $u_{0}$ in $W_{0}^{1, p}(\Omega)$ because $I$ satisfies the bounded Palais-Smale condition. On the other hand, Lemma 33 guarantees that $u_{0} \neq 0$ (note $\left.\sup _{n \geq n_{0}} I_{+n}\left(u_{n}\right) \leq 0\right)$. Therefore $u_{0}$ is a nontrivial critical point of $I$.

Proof of Theorem 31(iv). Let $n_{0} \in \mathbb{N}$ be such that $\alpha-p / n_{0}>\lambda_{1}\left(a_{\infty}\right)$. Applying Lemma 30 to $f_{-n}$ for $n \geq n_{0}$ (and since $\alpha_{0}-p / n<\lambda_{1}\left(a_{0}\right)$ ), we can choose $q_{0} \in\left(p, p^{*}\right]$ and $\rho_{n}>0$ such that $\delta_{n}:=\inf \left\{I_{-n}(u):\|u\|_{q_{0}}=\rho_{n}\right\}>0$. By noting that $f_{-n}(x, t) / t^{p-1} \rightarrow \alpha-p / n>\lambda_{1}\left(a_{\infty}\right)$ as $t \rightarrow+\infty$ for every $n \geq n_{0}$, and applying Lemma 28 to $f_{-n}$ instead of $f$, we see that $I_{-n}$ satisfies the Palais-Smale condition. Therefore, the proof of Theorem 25(ii) implies that, for every $n \geq n_{0}$, there exists 
a critical point $u_{n} \in W_{0}^{1, p}(\Omega)$ of $I_{-n}$ such that $I_{-n}\left(u_{n}\right) \geq \delta_{n}>0$. By Lemma 32, $\left\{u_{n}\right\}$ is bounded in $W_{0}^{1, p}(\Omega)$. Thus, by arguing as in case (i), we find a subsequence $\left\{u_{n}\right\}$ converging to some $u_{0}$ in $W_{0}^{1, p}(\Omega)$. Also, Lemma 33 yields $u_{0} \neq 0$ (note that $\inf _{n \geq n_{0}} I_{-n}\left(u_{n}\right) \geq 0$ ). This shows that $u_{0}$ is a nontrivial critical point of $I$.

The doubly resonant case. Choose smooth nonnegative functions $\varphi$ and $\psi$ on $[0,+\infty)$ satisfying $\varphi(t)=1$ if $0 \leq t \leq 2, \varphi(t)=0$ if $t \geq 4, \psi(t)=0$ if $t \leq 5$, and $\psi(t)=1$ if $t \geq 10$. Define approximate functionals on $W_{0}^{1, p}(\Omega)$ by

$$
\tilde{I}_{ \pm n}(u):=I(u) \mp \frac{1}{n} \psi\left(\|u\|_{p}^{p}\right)\left\|u_{+}\right\|_{p}^{p} \pm \frac{1}{n} \varphi\left(\|u\|_{p}^{p}\right)\left\|u_{+}\right\|_{p}^{p} .
$$

Because $\tilde{I}_{ \pm n}(u)=I_{\mp n}(u)$ provided $\|u\|_{p} \leq 2$, the following result can be proved by the same argument as in Lemma 33. We omit the proof.

Lemma 34. Assume $(\mathrm{H} 0-)$ or $(\mathrm{HF} 0-)$ (resp. $(\mathrm{H} 0+)$ or $(\mathrm{HF} 0+))$. Let $u_{n} \neq 0$ be an element of $W_{0}^{1, p}(\Omega)$ satisfying $\left(\tilde{I}_{ \pm n}\right)^{\prime}\left(u_{n}\right)=0$ for every $n \in \mathbb{N}$ and $\inf _{n} \tilde{I}_{ \pm n}\left(u_{n}\right) \geq 0$ (resp. $\left.\sup _{n} \tilde{I}_{ \pm n}\left(u_{n}\right) \leq 0\right)$. Then $\liminf _{n \rightarrow \infty}\left\|u_{n}\right\|_{p}>0$.

Lemma 35. If $\alpha \pm p / n \neq \lambda_{1}\left(a_{\infty}\right)$, then $\tilde{I}_{ \pm n}$ (with the matching sign) satisfies the Palais-Smale condition.

Proof. Let $\left\{u_{m}\right\}$ be a Palais-Smale sequence of $\tilde{I}_{+n}$ or $\tilde{I}_{-n}$. If $\left\|u_{m}\right\|_{p} \rightarrow \infty$ occurs, then $\tilde{I}_{ \pm n}\left(u_{m}\right)=I_{ \pm n}\left(u_{m}\right)$ for sufficiently large $m$. So, by applying Lemma 28 to $f_{ \pm n}$ (note that $\alpha \pm p / n \neq \lambda_{1}\left(a_{\infty}\right)$ ), we have a contradiction if $\left\|u_{m}\right\|_{p} \rightarrow \infty$. Consequently, we see that $\left\|u_{m}\right\|_{p}$ is bounded. Then, by the same reason as in Lemma 28, $\left\{u_{m}\right\}$ has a convergent subsequence in $W_{0}^{1, p}(\Omega)$.

Because $\tilde{I}_{ \pm n}(u)=I_{ \pm n}(u)$ provided $\|u\|_{p} \geq 10$, the following result can be proved by the same argument as in Lemma 32. We omit the proof.

Lemma 36. If either $(\mathrm{H}+)$ or $(\mathrm{HF}+)$ (resp. either $(\mathrm{H}-)$ or $(\mathrm{HF}-))$ and $\left\{u_{n}\right\}$ satisfies

$$
\begin{array}{rll} 
& \sup _{n \in \mathbb{N}} \tilde{I}_{ \pm n}\left(u_{n}\right)<+\infty \quad \text { and } \quad \lim _{n \rightarrow \infty}\left\|\left(\tilde{I}_{ \pm n}\right)^{\prime}\left(u_{n}\right)\right\|_{W_{0}^{1, p}(\Omega)^{*}}=0 \\
\text { (resp. } & \left.\inf _{n \in \mathbb{N}} \tilde{I}_{ \pm n}\left(u_{n}\right)>-\infty \quad \text { and } \quad \lim _{n \rightarrow \infty}\left\|\left(\tilde{I}_{ \pm n}\right)^{\prime}\left(u_{n}\right)\right\|_{W_{0}^{1, p}(\Omega)^{*}}=0\right),
\end{array}
$$

$\left\{u_{n}\right\}$ is bounded in $W_{0}^{1, p}(\Omega)$.

Proof of Theorem $31(\mathrm{v})$. Note that $\tilde{I}_{-n}(u)=I_{-n}(u)$ provided $\|u\|_{p} \geq 10$ and $\tilde{I}_{-n}(u)=I_{+n}(u)$ if $\|u\|_{p} \leq 2$. So, by a similar argument to that in (i), $\tilde{I}_{-n}$ has a global minimizer $u_{n}$. Moreover, by a similar argument to that in (iii) (note that $f_{+n}(x, t) / t^{p-1} \rightarrow \alpha_{0}+p / n>\lambda_{1}\left(a_{0}\right)$ as $t \rightarrow+0$ and $f_{-n}(x, t) / t^{p-1} \rightarrow \alpha-p / n<$ $\lambda_{1}\left(a_{\infty}\right)$ as $\left.t \rightarrow+\infty\right)$, we have $\tilde{I}_{-n}\left(u_{n}\right)<0$, whence $u_{n} \neq 0$. Because Lemma 36 implies the boundedness of $\left\|u_{n}\right\|$, by the same argument as in (i), we see that $\left\{u_{n}\right\}$ 
is a bounded Palais-Smale sequence of $I$. Therefore, we may assume that $u_{n}$ converges to some $u_{0}$ in $W_{0}^{1, p}(\Omega)$ by choosing a subsequence. On the other hand, Lemma 33 yields $\liminf _{n \rightarrow \infty}\left\|u_{n}\right\|_{p}>0$. Hence $u_{0} \neq 0$. This means that $u_{0}$ is a nontrivial critical point of $I$.

Proof if Theorem 31(vi). Note that $\tilde{I}_{+n}(u)=I_{+n}(u)$ provided $\|u\|_{p} \geq 10$ and $\tilde{I}_{+n}(u)=I_{-n}(u)$ if $\|u\|_{p} \leq 2$. So, because $f_{-n}(x, t) / t^{p-1} \rightarrow \alpha_{0}-p / n<\lambda_{1}\left(a_{0}\right)$ as $t \rightarrow+0$ and $f_{+n}(x, t) / t^{p-1} \rightarrow \alpha+p / n>\lambda_{1}\left(a_{\infty}\right)$ as $t \rightarrow+\infty$, by a similar argument to those in (ii) and (iv), for each $n$, we have a nontrivial critical point $u_{n}$ of $\tilde{I}_{+n}$ with $\tilde{I}_{+n}\left(u_{n}\right)>0$. As a result, by a similar reasoning as in (v), we can obtain a nontrivial critical point of $I$.

\section{Acknowledgements}

The second author would like to express her sincere thanks to Professor Shizuo Miyajima for helpful comments and encouragement.

\section{References}

[Anane 1987] A. Anane, Etude des valeurs propres et de la résonnance pour l'opérateur p-Laplacien, Ph.D. thesis, Université Libre de Bruxelles, 1987.

[Cuesta et al. 1999] M. Cuesta, D. de Figueiredo, and J.-P. Gossez, "The beginning of the Fučik spectrum for the p-Laplacian”, J. Differential Equations 159:1 (1999), 212-238. MR 2001f:35308 Zbl 0947.35068

[Damascelli 1998] L. Damascelli, "Comparison theorems for some quasilinear degenerate elliptic operators and applications to symmetry and monotonicity results", Ann. Inst. H. Poincaré Anal. Non Linéaire 15:4 (1998), 493-516. MR 99e:35081 Zbl 0911.35009

[Dancer and Perera 2001] N. Dancer and K. Perera, "Some remarks on the Fučik spectrum of the p-Laplacian and critical groups", J. Math. Anal. Appl. 254:1 (2001), 164-177. MR 2001k:35238 Zbl 0970.35056

[Deimling 1985] K. Deimling, Nonlinear functional analysis, Springer, Berlin, 1985. MR 86j:47001 Zbl 0559.47040

[Fukagai and Narukawa 2007] N. Fukagai and K. Narukawa, "On the existence of multiple positive solutions of quasilinear elliptic eigenvalue problems", Ann. Mat. Pura Appl. (4) 186:3 (2007), 539-564. MR 2008d:35052 Zbl 1223.35132

[García-Huidobro et al. 1995] M. García-Huidobro, R. Manásevich, and P. Ubilla, "Existence of positive solutions for some Dirichlet problems with an asymptotically homogeneous operator", Electron. J. Differential Equations 1995:10 (1995), 1-22. MR 96f:35053 Zbl 0823.35057

[Kim 2009] Y.-H. Kim, "A global bifurcation for nonlinear equations with nonhomogeneous part", Nonlinear Anal. 71:12 (2009), 738-743. MR 2011d:35048 Zbl 1238.35009

[Kim and Kim 2010] I.-S. Kim and Y.-H. Kim, "Global bifurcation for equations involving nonhomogeneous operators in an unbounded domain", Nonlinear Anal. 73:4 (2010), 1057-1064. MR 2011e:35023 Zbl 1194.35492

[Kyritsi et al. 2010] S. T. Kyritsi, D. O'Regan, and N. S. Papageorgiou, "Existence of multiple solutions for nonlinear Dirichlet problems with a nonhomogeneous differential operator", Adv. Nonlinear Stud. 10:3 (2010), 631-657. MR 2011e:35116 Zbl 1216.35037 
[Lieberman 1988] G. M. Lieberman, "Boundary regularity for solutions of degenerate elliptic equations”, Nonlinear Anal. 12:11 (1988), 1203-1219. MR 90a:35098 Zbl 0675.35042

[Mawhin and Willem 1989] J. Mawhin and M. Willem, Critical point theory and Hamiltonian systems, Applied Mathematical Sciences 74, Springer, New York, 1989. MR 90e:58016 Zbl 0676.58017

[Miyajima et al. 2012] S. Miyajima, D. Motreanu, and M. Tanaka, "Multiple existence results of solutions for the Neumann problems via super- and sub-solutions", J. Funct. Anal. 262:4 (2012), 1921-1953. MR 2873865 Zbl pre06012152

[Motreanu and Papageorgiou 2011] D. Motreanu and N. S. Papageorgiou, "Multiple solutions for nonlinear Neumann problems driven by a nonhomogeneous differential operator", Proc. Amer. Math. Soc. 139:10 (2011), 3527-3535. MR 2012c:35166 Zbl 1226.35021

[Motreanu et al. 2011] D. Motreanu, V. V. Motreanu, and N. S. Papageorgiou, "Multiple constant sign and nodal solutions for nonlinear Neumann eigenvalue problems", Ann. Sc. Norm. Super. Pisa Cl. Sci. (5) 10:3 (2011), 729-755. MR 2012m:35087 Zbl 1234.35169

[Prado and Ubilla 1998] H. Prado and P. Ubilla, "Existence of nonnegative solutions for generalized p-Laplacians", pp. 289-298 in Reaction diffusion systems (Trieste, 1995), edited by G. Caristi and E. Mitidieri, Lecture Notes in Pure and Appl. Math. 194, Dekker, New York, 1998. MR 98j:35069 Zbl 0913.35044

[Robinson 2004] S. B. Robinson, "On the second eigenvalue for nonhomogeneous quasi-linear operators”, SIAM J. Math. Anal. 35:5 (2004), 1241-1249. MR 2005a:35219 Zbl 1061.35071

[Tanaka 2009] M. Tanaka, "Existence of constant sign solutions for the $p$-Laplacian problems in the resonant case with respect to Fučik spectrum", SUT J. Math. 45:2 (2009), 149-166. MR 2011d:35207 Zbl 1196.35115

[Tanaka 2012a] M. Tanaka, "The antimaximum principle and the existence of a solution for the generalized $p$-Laplace equations with indefinite weight”, Differ. Equ. Appl. 4:4 (2012), 581-613.

[Tanaka 2012b] M. Tanaka, "Existence of the Fučik type spectrums for the generalized $p$-Laplace operators”, Nonlinear Anal. 75:7 (2012), 3407-3435. MR 2891177 Zbl 1241.35091

Received June 19, 2012.

\section{DUMITRU MOTREANU}

DÉPARTMENT DE MATHÉMATIQUES

UNIVERSITÉ DE PERPIGNAN

52 avenue Paul Alduy

66860 PERPIGNAN

FRANCE

motreanu@univ-perp.fr

MIEKO TANAKA

DEPARTMENT OF MATHEMATICS

TOKYO UNIVERSITY OF SCIENCE

KAGURAZAKA 1-3

SHINJYUKU-KU

TOKYO 162-8601

JAPAN

tanaka@ma.kagu.tus.ac.jp 


\title{
PACIFIC JOURNAL OF MATHEMATICS
}

\author{
msp.org/pjm
}

Founded in 1951 by E. F. Beckenbach (1906-1982) and F. Wolf (1904-1989)

\section{EDITORS}

V. S. Varadarajan (Managing Editor)

Department of Mathematics

University of California

Los Angeles, CA 90095-1555

pacific@math.ucla.edu

Paul Balmer

Department of Mathematics

University of California

Los Angeles, CA 90095-1555

balmer@math.ucla.edu

Daryl Cooper

Department of Mathematics

University of California

Santa Barbara, CA 93106-3080 cooper@math.ucsb.edu

Jiang-Hua $\mathrm{Lu}$

Department of Mathematics

Pokfulam Rd., Hong Kong jhlu@maths.hku.hk
The University of Hong Kong

Don Blasius

Department of Mathematics University of California

Los Angeles, CA 90095-1555

blasius@math.ucla.edu

Robert Finn

Department of Mathematics Stanford University

Stanford, CA 94305-2125

finn@math.stanford.edu

Sorin Popa

Department of Mathematics

University of California

Los Angeles, CA 90095-1555 popa@math.ucla.edu

Paul Yang

Department of Mathematics Princeton University

Princeton NJ 08544-1000

yang@math.princeton.edu

\section{PRODUCTION}

Silvio Levy, Scientific Editor, production@msp.org

\section{SUPPORTING INSTITUTIONS}

ACADEMIA SINICA, TAIPEI

CALIFORNIA INST. OF TECHNOLOGY

INST. DE MATEMÁTICA PURA E APLICADA

KEIO UNIVERSITY

MATH. SCIENCES RESEARCH INSTITUTE

NEW MEXICO STATE UNIV.

OREGON STATE UNIV.

\author{
STANFORD UNIVERSITY \\ UNIV. OF BRITISH COLUMBIA \\ UNIV. OF CALIFORNIA, BERKELEY \\ UNIV. OF CALIFORNIA, DAVIS \\ UNIV. OF CALIFORNIA, LOS ANGELES \\ UNIV. OF CALIFORNIA, RIVERSIDE \\ UNIV. OF CALIFORNIA, SAN DIEGO \\ UNIV. OF CALIF., SANTA BARBARA
}

\author{
Vyjayanthi Chari \\ Department of Mathematics \\ University of California \\ Riverside, CA 92521-0135 \\ chari@math.ucr.edu \\ Kefeng Liu \\ Department of Mathematics \\ University of California \\ Los Angeles, CA 90095-1555 \\ liu@math.ucla.edu \\ Jie Qing \\ Department of Mathematics \\ University of California \\ Santa Cruz, CA 95064 \\ qing@cats.ucsc.edu
}

These supporting institutions contribute to the cost of publication of this Journal, but they are not owners or publishers and have no responsibility for its contents or policies.

See inside back cover or msp.org/pjm for submission instructions.

The subscription price for 2013 is US \$400/year for the electronic version, and \$485/year for print and electronic.

Subscriptions, requests for back issues and changes of subscribers address should be sent to Pacific Journal of Mathematics, P.O. Box 4163, Berkeley, CA 94704-0163, U.S.A. The Pacific Journal of Mathematics is indexed by Mathematical Reviews, Zentralblatt MATH, PASCAL CNRS Index, Referativnyi Zhurnal, Current Mathematical Publications and the Science Citation Index.

The Pacific Journal of Mathematics (ISSN 0030-8730) at the University of California, c/o Department of Mathematics, 798 Evans Hall \#3840, Berkeley, CA 94720-3840, is published twelve times a year. Periodical rate postage paid at Berkeley, CA 94704, and additional mailing offices. POSTMASTER: send address changes to Pacific Journal of Mathematics, P.O. Box 4163, Berkeley, CA 94704-0163.

PJM peer review and production are managed by EditFLOW ${ }^{\circledR}$ from Mathematical Sciences Publishers.

\section{PUBLISHED BY}

mathematical sciences publishers

nonprofit scientific publishing

http://msp.org/

(C) 2013 Mathematical Sciences Publishers 


\section{PACIFIC JOURNAL OF MATHEMATICS}

Volume $265 \quad$ No. $1 \quad$ September 2013

Genus-two Goeritz groups of lens spaces

SANGBUM CHO

A compact embedding theorem for generalized Sobolev spaces

SENG-KeE ChUa, SCOTT Rodney and Richard L. WheEden

Partial integrability of almost complex structures and the existence of

solutions for quasilinear Cauchy-Riemann equations

CHONG-KYU HAN and JONG-DO PARK

An overdetermined problem in potential theory

DMITRY KHAVINSON, ERIK LUNDBERG and RAZVAN TEODORESCU

Quasisymmetric homeomorphisms on reducible Carnot groups

XIANGDONG XIE

Capillarity and Archimedes' principle

JOHN MCCUAN and RAY TREINEN

Generalized eigenvalue problems of nonhomogeneous elliptic operators and their application

DUmitru Motreanu and Mieko TANaKa

Weighted Ricci curvature estimates for Hilbert and Funk geometries

SHIN-ICHI OHTA

On generalized weighted Hilbert matrices

EMMANUEL PREISSMANN and OLIVIER LÉVÊQUE

Unique prime decomposition results for factors coming from wreath product 221 groups

J. OWEN SizEMORE and ADAM WinCHESTER

On volume growth of gradient steady Ricci solitons

Guofang Wei and Peng Wu

Classification of moduli spaces of arrangements of nine projective lines

FEI YE 